\title{
Echo statistics of individual and aggregations of scatterers in the water column of a random, oceanic waveguide
}

\author{
Benjamin A. Jones ${ }^{\text {a) }}$ and John A. Colosi \\ Oceanography Department, Naval Postgraduate School, 833 Dyer Road, Monterey, California 93943 \\ Timothy K. Stanton \\ Department of Applied Ocean Physics and Engineering, Woods Hole Oceanographic Institution, MS \#11, \\ Woods Hole, Massachusetts 02543
}

(Received 20 June 2013; revised 9 April 2014; accepted 16 May 2014)

\begin{abstract}
The relative contributions of various physical factors to producing non-Rayleigh distributions of echo magnitudes in a waveguide are examined. Factors that are considered include (1) a stochastic, range-dependent sound-speed profile, (2) a directional acoustic source, (3) a variable scattering response, and (4) an extended scattering volume. A two-way parabolic equation model, coupled with a stochastic internal wave model, produces realistic simulations of acoustic propagation through a complex oceanic sound speed field. Simulations are conducted for a single frequency $(3 \mathrm{kHz})$, monostatic sonar with a narrow beam $\left(5^{\circ}-3 \mathrm{~dB}\right.$ beam width). The randomization of the waveguide, range of propagation, directionality of the sonar, and spatial extent of the scatterers each contribute to the degree to which the echo statistics are non-Rayleigh. Of critical importance are the deterministic and stochastic processes that induce multipath and drive the one-way acoustic pressure field to saturation (i.e., complex-Gaussian statistics). In this limit predictable statistics of echo envelopes are obtained at all ranges. A computationally low-budget phasor summation can successfully predict the probability density functions when the beam pattern and number of scatterers ensonified are known quantities. [http://dx.doi.org/10.1121/1.4881925]
\end{abstract}

PACS number(s): 43.30.Gv, 43.30.Ft [APL]

Pages: 90-108

\section{INTRODUCTION}

The physical complexities of a shallow water environment directly impact active acoustic measurement systems including both military and scientific sonar systems. An understanding of how this complex environment affects echo statistics can aid operators in predicting sonar performance, lead to more realistic simulations for use in training sonar operators, and help researchers develop automated discriminators that could filter clutter (i.e., non-target echoes) from echoes of interest in sonar data. In addition to military applications, the interpretation of echoes from aggregations of scatterers observed on horizontal-looking sonar systems has applications to biologic surveys, ${ }^{1-6}$ where species discrimination and abundance estimation are of interest. Understanding the acoustic response of each of the various complexities that exist in shallow water is crucial to predicting and interpreting echo statistics in these regions. The intent of this study is to quantify the effects of various environmental, sonar system, and scatterer characteristics on the statistics of long-range (i.e., much greater than the water depth) echoes from scatterers in the water column.

For mid-frequency (i.e., 1 to $9 \mathrm{kHz}$ ) horizontal-looking sonar systems operating at long-ranges, the ocean is a waveguide trapping sound energy between the pressure release boundary at the surface and a penetrable bottom. The waveguide is characterized by many factors that can be

\footnotetext{
${ }^{\text {a) }}$ Author to whom correspondence should be addressed. Electronic mail: bajones@nps.edu
}

subdivided into deterministic and stochastic factors. To first order, the deterministic factors affecting the echo statistics are: (1) The average water column sound-speed profile (SSP) and (2) large-scale bathymetric features. Stochastic factors can include (1) sound-speed perturbations in the water column [such as those caused by internal waves (IWs)], (2) small-scale bottom roughness, and (3) surface roughness.

An extensive amount of work has been conducted on model development of acoustic scattering by compact objects in an ocean waveguide including the widely-used normalmode-,${ }^{7,8}$ transition-matrix-, ${ }^{9-12}$ and parabolic-equation ${ }^{13}$ (PE)-based approaches. Much of the analysis associated with waveguide scattering has focused on scattering by an individual or a few compact shapes in deterministic waveguides of varying levels of complexity including: Homogeneous waveguides, ${ }^{14-16}$ plane-layered (rangeindependent) waveguides, ${ }^{7,8,10,17-20}$ depth-dependent water column properties, ${ }^{11,21}$ and homogeneous water column properties with range-dependent boundaries. ${ }^{18,21,22}$ The importance of factors such as refractive effects of the waveguide and a range-dependent bottom are illustrated in Ref. 21 where, for example, echo levels are shown to vary by up to $10 \mathrm{~dB}$ at $20 \mathrm{~km}$ in a refractive (depth-dependent) environment compared with a homogeneous water column for a vertically extended scatterer.

A further complexity of the waveguide, that of fluctuating material properties, has long been appreciated with respect to one-way propagation (e.g., see Refs. 23 and 24). However, the effects of a fluctuating waveguide on echo 
statistics has garnered attention only more recently. ${ }^{25,26}$ Andrews et al. investigate multiple scattering and attenuation by aggregations of fish in a random waveguide in terms of both the magnitudes of these effects and the regimes where they may be significant. ${ }^{25}$ Notably, these studies model the sound speed fluctuations as a random draw of observed SSPs rather than incorporating a coherent sound speed field-an advance incorporated in this study.

In addition to the waveguide, characteristics of the acoustic system (i.e., the source and receiver) can affect the echo statistics. For a broadband system, the range resolution and, hence, the number of scatterers ensonified, is a function of bandwidth. Since the number of scatterers simultaneously ensonified affects the echo statistics, these statistics are a function of bandwidth. Source and receiver beam patterns (BPs) can also be important factors. In the case of scatterers randomly located in the main lobe of a directional acoustic beam, the BP value applied to each scatterer becomes a statistical quantity. This has been shown to have a significant effect on the distribution of echo envelopes (i.e., the magnitude of the complex pressure at the receiver) for scatterers in a direct-path geometry. ${ }^{27}$ Furthermore, in a study on boundary reverberation for a horizontal-looking sonar system, LePage predicts a shift in the shape parameter of the $K$ distribution (away from Rayleigh) associated with the beamforming process of a vertical receiver array. ${ }^{28}$ The influence of a narrow BP (in both elevation and azimuth) on the distribution of echo envelopes is a first-order effect not believed to have been previously quantified for water-column scatterers ensonified in a waveguide.

Finally, the scatterer itself can have several characteristics, both deterministic and stochastic, that affect the echo statistics. Characteristics such as shape and location may be well known (e.g., a rock outcropping) or may be highly variable or even random in time and space (e.g., a mobile school of fish with a time-varying morphology). ${ }^{29}$ Depending on the ratio of the acoustic wavelength to the scatterer size and the scatterer shape, the scattering object may have a directional BP (e.g., large fish or marine mammals at high frequencies) or an isotropic point-scatterer-like BP (e.g., swim bladderbearing fish at frequencies near resonance). In the former case, the degree to which the scatterer is randomly oriented will directly affect the echo statistics. ${ }^{30}$ The scattering response may also have a stochastic component related to the size and distribution of scatterers simultaneously ensonified. This factor is a function of the volume of the sonar resolution cell and the number of individual scattering objects within that cell. For example, the echo from a single object with fixed orientation may have a delta-function probability density function (PDF); whereas, the echo from many closely spaced scatterers of similar scattering amplitude, but random phase, would tend toward Rayleigh-distributed envelopes. Finally, the spatial extent of the scatterer may affect the echo statistics depending on the degree to which it spans different parts of the waveguide as demonstrated in this article.

In this study, echo statistics from aggregations of scatterers located in the water column of an oceanic waveguide are analyzed over a wide range of conditions through both numerical and analytical methods. The scatterers are ensonified by long-range directional mid-frequency sonar. The various effects associated with the waveguide, sonar, and scatterers are studied both in isolation and collectively, namely (1) the source directionality and BP, (2) the depthdependent SSP, (3) random sound-speed perturbations like those caused by IWs, (4) the waveguide response, and (5) the acoustic response and spatial distribution of the scatterer itself. Although many complexities are not accounted for (e.g., broadband waveforms, surface roughness, etc.), this analysis provides a basis for the interpretation of observations and for evaluating other physical and system factors that might affect the echo statistics.

In contrast to Refs. 25 and 26 which focus on the first two moments of intensity statistics (key to determining abundance from acoustic assessments of fish stocks), the interest here extends to the higher-order moments of amplitude statistics. Echo magnitude PDFs calculated in this study, and particularly the high amplitude, low probability tail of these distributions, directly apply to probabilities of false alarm in target detection calculations. These PDFs are compared, in some key cases, with the $K$ distribution, an empirically validated method commonly used in describing echo statistics in long-range sonar applications, particularly bottom reverberation (e.g., see Ref. 31 and references within).

Advances in the approach presented here are threefold. First, echo statistics are examined in a fluctuating waveguide where the sound speed perturbations are modeled as an empirically-based, spatially coherent sound-speed gradient within a diffuse IW field. This portrays the smooth transition of waveguide properties in both depth and range in contrast to Ref. 25 which involves a random draw of observed SSPs. Second, the effects on echo magnitude PDFs of a narrow BP in both azimuth and elevation are analyzed and predictions are made with a simple numerical model. This is an effect that had been quantified, previously, only for the direct path case. Finally, the effects on the echo distributions of varying the horizontal spatial extent of an aggregation in a stochastic waveguide are shown both analytically and through numerical calculations.

The primary simplifications incorporated in this research are the use of continuous wave (CW) theory and the assumption of first-order scattering with no extinction. The latter assumptions are made due to the narrow range of circumstances where higher-order scattering and extinction have been shown to be relevant. ${ }^{25,32}$ Specifically, for the region that inspired this modeling study, the numerical density of fish is low enough throughout the area that extinction is negligible and effects of multiple scattering are not significant. ${ }^{33}$ Finally, although this research does not explicitly treat frequency modulated sources, the first-order effects illustrated here are applicable to narrowband systems where the $\mathrm{CW}$ assumption is applied to overlapping echoes received within the inverse bandwidth of the signal. ${ }^{34}$

This article is organized as follows. Section II describes theory and applications for four analytic models of echo magnitude PDFs used in this study. Section III describes a two-way PE model and the method by which IW-induced sound-speed perturbations are incorporated into that model. Additionally, this section describes a phasor summation (PS) 
method used to generate echo statistics in this study. Echo statistics of scatterers randomly located in depth in a simple, deterministic waveguide are described in Sec. IV, while statistics of echoes in a waveguide with IWs is presented in Sec. V. The issue of thin layers of scatterers in a waveguide with IWs is discussed in Sec. VI. Sections VII and VIII have discussion and concluding remarks, respectively.

\section{PDFS ASSOCIATED WITH ACOUSTIC BACKSCATTER}

Many acoustic reverberation studies have focused on identifying candidate analytical PDFs to characterize echoes from objects in the water column. An echo PDF can be sensitive to characteristics of the scatterer, the environment, the ensonifying system, and even processing techniques used to extract echoes from the data. In this paper comparisons are made between numerical model predictions and two distributions that have been commonly used to characterize sonar echoes: The Rayleigh and the $K$ distributions. Additionally, comparisons are made with analytical, physics-based echo PDFs for three general cases: (1) An arbitrary number of scatterers in a waveguide, (2) scatterers in a waveguide ensonified by a directional sonar-where waveguide effects are incorporated into an existing physics-based, direct-path theory, and (3) spatially extended aggregations of scatterers in a waveguide - a newly developed theory.

\section{A. Rayleigh distribution}

A zero-mean complex Gaussian distribution arises in the limit of an infinite sum of statistically independent signals with identically distributed amplitudes and phases that are uniformly distributed over the interval $2 \pi$. In this limit the resultant field is often termed saturated where the signal envelope is Rayleigh distributed yielding a scintillation index (SI) value, according to Eq. (B1), of 1. A Rayleigh $\mathrm{PDF}$ is a single parameter distribution of the form

$$
\mathrm{p}_{X}(x)=\frac{x}{h^{2}} e^{-x^{2} / 2 h^{2}}, \quad x \geq 0,
$$

where $\mathrm{p}(\cdot)$ indicates the PDF of the subscripted (and capitalized) random variable over the values listed in parenthesis. The parameter $h$ is related to the root-mean-square (rms) value by the relation $\left\langle X^{2}\right\rangle^{1 / 2}=\sqrt{2} h$. This distribution was observed in early studies of volume reverberation where the echo envelopes of a high frequency acoustic system were Rayleigh distributed. ${ }^{35}$ For a military sonar system the Rayleigh distribution has become the benchmark for determining the likelihood that a scatterer (or collection of scatterers) will cause a false-alarm. Echoes from non-targets that have an envelope PDF with an elevated, high amplitude tail when compared with this distribution can be considered clutter. ${ }^{36,37}$ Similarly, in scientific studies, deviations from the Rayleigh distribution contain important information on the scatterer of interest. ${ }^{38}$ All echo envelope distributions modeled in this study are, therefore, compared with a Rayleigh distribution to measure the contribution of specific parameters toward any deviation from this benchmark.

\section{B. K distribution}

The $K$ distribution has been widely used in both radar and sonar theory for describing echo envelopes. This distribution has been used to model the high amplitude tail of PDFs of echoes envelopes from seafloor reverberation as well as shipwrecks. ${ }^{31,39}$ This two-parameter PDF is given by

$$
\mathrm{p}_{X}(x)=\frac{4}{\sqrt{\lambda_{k}} \Gamma\left(\alpha_{k}\right)}\left(\frac{x}{\sqrt{\lambda_{k}}}\right)^{\alpha_{k}} K_{\alpha_{k}-1}\left(\frac{2 x}{\sqrt{\lambda_{k}}}\right), \quad x \geq 0,
$$

where $K_{\nu}$ is a modified Bessel function of the second kind, $\Gamma$ is a Gamma function, and the two free parameters are $\alpha_{k}$, a shape parameter, and $\lambda_{k}$, a scale parameter. The shape parameter, which is related to the number of scatterers in the beam, ${ }^{40}$ has often been used as a proxy for the likelihood of a false alarm. As the shape parameter increases, the $K$ distribution tends toward a Rayleigh distribution. A low shape parameter represents a higher probability of false alarm.

\section{Aggregation of scatterers with random phases in a frozen waveguide (no beam pattern)}

It is known that when the magnitude of a signal is Rayleigh distributed the PDF of the intensity will follow an exponential distribution (i.e., a chi-square distribution with two-degrees of freedom). ${ }^{41}$ However, an exponential distribution can also arise in the echo envelopes (i.e., the square root of intensity) associated with a single scatterer in a random waveguide as will be shown in this section. Consider the case of a monostatic, omni-directional sonar ensonifying an isotropic (monopole) scatterer. In this case, under the frozen ocean approximation where the waveguide does not change during the propagation of the signal, the pressure at the receiver is expected to be proportional to the square of the pressure at the scatterer due to reciprocity

$$
p_{r} \propto p_{s}^{2}
$$

Given a known statistical nature of the pressure field at the scatterer location, this relationship makes it possible to deduce the statistics at the receiver for some simple cases. For a random location in depth or delay time the pressure field at some scatterer can be represented as a complex random variable

$$
P_{s}=X+\mathrm{i} Y
$$

with an envelope of $E_{s}=\sqrt{X^{2}+Y^{2}}$. For the case of $N$ interfering scatterers with fixed scattering responses, Eq. (4) can be substituted into Eq. (3) and summed over all contributing scatterers to give the pressure at a receiver of a monostatic system

$$
P_{r}=\sum_{n=1}^{N}\left(X_{n}+\mathrm{i} Y_{n}\right)^{2}
$$


The envelope at the receiver is then given as

$$
E_{r}=\left[\left(\sum_{n=1}^{N} X_{n}^{2}-Y_{n}^{2}\right)^{2}+\left(\sum_{n=1}^{N} 2 X_{n} Y_{n}\right)^{2}\right]^{(1 / 2)}
$$

which conveniently reduces, for the case of $N=1$, to

$$
E_{r}=X^{2}+Y^{2}=E_{s}^{2} .
$$

An important application of this result is in the case of a single scatterer located in a waveguide. At some range, $r_{\text {sat }}$, it is assumed that the pressure field becomes saturated by interference of the forward-scattered acoustic wave. At this range the real and complex parts of the pressure field, $X$ and $Y$, are uncorrelated Gaussian random variables and the envelope, $E_{s}$, is Rayleigh distributed. According to Eq. (7), then, the envelope at the receiver is the square of a Rayleigh random variable which is an exponential random variable with a PDF given by

$$
\mathrm{p}_{E_{r}}(x)=\frac{1}{\beta} e^{-x / \beta}, \quad x \geq 0,
$$

where $\beta=\left\langle E_{r}\right\rangle$.

\section{Aggregation of scatterers in a directional sonar beam (direct path and waveguide cases)}

Combining the work of Ehrenberg et $a l^{42,43}$ and Barakat, ${ }^{44}$ Chu and Stanton ${ }^{27}$ provide an analytical model for the echo statistics of a finite number of isotropic scatterers, each with an arbitrary scattering response and each randomly located within a directional sonar beam [see Eqs. (2), (3), and (19) of Ref. 27]. In contrast to the $K$ distribution, in which the parameters must be estimated from observations (see, for example, Abraham and Lyons), ${ }^{45}$ the Chu-Stanton theory is physics-based and, therefore, predictive given some knowledge about the sonar system and the scatterers. This theory was developed for the case of a direct-path environment in which echoes from boundaries (sea surface and seafloor) do not overlap with the echoes of interest.

This theory has shown that, under important conditions, the BP will cause a strongly non-Rayleigh echo PDF. These conditions include the case of a narrow BP or few scatterers even when the scatterer itself has a Rayleigh-distributed scattering response. In the present study it is seen that BP effects are important under similar conditions in a waveguide and that the Chu-Stanton theory can be applied in this case with two key modifications. First, the distribution of BP values for a random position of the scatterer in the beam, $\left[p_{B}\right.$ of Eq. (19) in Ref. 27], is determined for a uniform distribution of positions that is limited to a narrow, nonsingular range in depression-elevation (DE) and the full range in azimuth. This "BP PDF," which can be determined numerically, accounts for the preferential propagation of low-angle energy in the waveguide, which is subject to less attenuation from interactions with the seafloor than energy radiated at high angles from the source. Second, the PDF of the envelope of the scattering response for each scatterer $\left[\mathrm{p}_{s_{n}}\right.$ of Eq. (19) in Ref. 27] is replaced by $\mathrm{p}_{s-w}$, the envelope PDF for the combined scatterer and waveguide response, $\Upsilon_{n}$. The term $\Upsilon_{n}$ is discussed in more detail in Sec. III C when describing the phasor summation method with Eq. (16). For example, in the special case of a delta-function scatterer in a saturated waveguide, $\mathrm{p}_{s-w}$ is given by Eq. (8).

\section{E. Aggregations of scatterers with varying spatial extents in a frozen waveguide (no beam pattern)}

A spatially compact aggregation of a large number of scatterers, all with similar scattering amplitudes (e.g., a small school of uniformly sized fish), induces a free-field scattering response that, when taking the modulus, is Rayleigh distributed $^{38}$ and is termed here a Rayleigh scatterer. For direct path calculations, such a scatterer can be efficiently modeled by a single point scatterer with a Rayleigh distributed response (e.g., see Ref. 27). However, in a waveguide the echo at long ranges is sensitive to the spatial extent of the scatterer leading, in some cases, to a distribution of echo envelopes that is strongly non-Rayleigh before considering BP effects. An explanation of this sensitivity can be shown using a transfer function model for the echo distribution as described below.

Let the frequency response of the one-way path in the waveguide, when randomly sampled at some location, $\vec{R}$, be defined as a random variable in phasor notation as

$$
H(\mathrm{f}, \vec{R})=H_{0}(\mathrm{f}, \vec{R}) e^{\mathrm{i} \Phi(\mathrm{f}, \vec{R})},
$$

where both the slowly varying magnitude of the waveguide response, $H_{0}$, and the phase, $\Phi$, are dependent upon frequency, $\mathrm{f}$, and a location in the waveguide. The envelope of the echo as measured at the receiver, also a random variable, is then given as

$$
E_{r}=\left|s(\mathrm{f}) \sum_{n=1}^{N_{s}} H_{0}^{2}\left(\mathrm{f}, \vec{R}_{n}\right) e^{2 \mathrm{i} \Phi\left(\mathrm{f}, \vec{R}_{n}\right)}\right|,
$$

where $s(\mathrm{f})$ is the source amplitude and $N_{s}$ is a large number of point scatterers that contribute to a single Rayleigh scatterer. These point scatterers, each with the same fixed scattering response (unity in this case), are located randomly at positions $\vec{R}_{n}$ within some scattering volume centered at $\vec{R}_{v}$. The waveguide response has been squared based on reciprocity of the acoustic paths for an omnidirectional source and a point scatterer and assuming the waveguide is fixed during the period of propagation as discussed in Sec. II C.

When the scattering volume is small compared with some correlation length of the waveguide intensity field, $H_{0}$ is considered independent of the location within the scattering volume yielding

$$
E_{r} \simeq s(\mathrm{f}) H_{0}^{2}\left(\mathrm{f}, \vec{R}_{v}\right)\left|\sum_{n=1}^{N_{s}} e^{2 \mathrm{i} \Phi\left(\mathrm{f}, \vec{R}_{n}\right)}\right| .
$$

Assuming the scattering volume is at least one-half of an acoustic wavelength in size, so that $\Phi$ is uniformly distributed over an interval of at least $\pi$, the magnitude of the summation is a Rayleigh random variable by the central limit theorem (CLT). Then, for the case of a complex-Gaussian distributed pressure field (i.e., a saturated environment) 
where $H_{0}^{2}$ is exponentially distributed, as shown in Sec. II C, the envelopes of the echoes are characterized by the distribution of the product of an exponential random variable and a Rayleigh random variable. However, when the scattering volume is large, $H_{0}$ cannot be considered independent of the location of each point scatterer. Then, in the case of a saturated environment, the summation in Eq. (10) is over $N_{s}$ independent and identically distributed random variables, $H^{2}$; and $E_{r}$ becomes a Rayleigh random variable by the CLT.

\section{NUMERICAL METHODS}

This study utilizes a two-way PE model and an empirically validated IW model for predicting echoes in a realistic oceanic environment. These models are used to calculate ensembles of echoes from which statistical distributions of the echoes can be drawn. Modeling comparisons are made with a computationally efficient phasor-summation based model also described in the section. Model validation is conducted, in part, by comparison with an analytical model developed for direct path calculations of echo envelope PDFs.

\section{A. Two-way parabolic equation model}

Predicting echo statistics in the ocean requires accounting for fluctuations in the waveguide and the scattering response, as well as the details of the sonar system. The approach taken here is to connect a range-dependent, twoway PE-based propagation model with a free-field, pointscatterer model. ${ }^{34}$ Unlike more sophisticated two-way PE models which calculate the backscattered component along the entire propagation path (see Ref. 47 and citations therein), this application uses a one-way PE model to and from the scatterer location. The numerical model, as implemented in this study, is a single frequency, monostatic, three-dimensional (3D) acoustic model using an $N \times 2 \mathrm{D}$ framework [i.e., a 3D environment modeled using $N$ twodimensional (2D) vertical slices at varying azimuthal angles from a source]. The major limitation of the $N \times 2 \mathrm{D}$ method is that out-of-plane propagation or scattering is not considered. It should be noted that the methods presented here can be extended to broadband, bistatic cases with only minor modification but with significant computational burden.

The model incorporates three major and somewhat independent components: (1) A PE algorithm for propagation, (2) a directional source field, and (3) a free-field scattering model. The propagation component of the model is a MATLAB-based derivative of the Range-dependent Acoustic Model. ${ }^{48}$ For two-way propagation, the "frozen ocean" approximation is made, where all environmental parameters are considered stationary for the two-way travel duration of the acoustic signal. The scattering model used in this study is a point scatterer or collection of point scatterers represented in the PE algorithm as one or more omnidirectional sources. This model is representative of scatterers that are small compared to a wavelength and that scatter sound isotropically (e.g., gas bubble near resonance. ${ }^{49}$ ) Finally, two types of starter fields are used as initial conditions for the propagation algorithm: (1) A directional field for the acoustic source and (2) an omnidirectional field at each scatterer. For the forward-propagation problem an arbitrary directional $\mathrm{BP}$ is modeled using the Fourier transform relationship between the wavenumber and spatial domains. ${ }^{34}$ The starter field for the back-propagation problem is modeled as a point scatterer by taking the complex output of the PE algorithm, $\psi\left(\mathrm{f} ; r_{s}, z_{s}\right)$, at the scatterer range, $r_{s}$, and depth, $z_{s}$, and applying a weighting function, $w$, to produce an omnidirectional BP,

$$
\psi\left(\mathrm{f} ; r_{0}, z\right)=\psi\left(\mathrm{f} ; r_{s}, z_{s}\right) F_{\mathrm{bs}} w(\mathrm{f} ; z) .
$$

The function $w(\mathrm{f} ; z)$ used in this application is the Greene's source, a weighted Gaussian function that is used as a starter field for PE calculations requiring good wide-angle properties. ${ }^{50}$ The backscattering amplitude, $F_{\mathrm{bs}}$, is either a constant (i.e., $F_{\mathrm{bs}} \equiv 1$ ) or a random variable drawn from a zero-mean complex Gaussian distribution. The latter case, with a Rayleigh distributed envelope, is used in cases of a Rayleigh scatterer.

\section{B. Internal wave model}

\section{Sound-speed perturbations due to diffuse IWs}

It is well known that diffuse, random fields of internal waves are present in the world's oceans, including stratified continental shelf regions, ${ }^{51}$ and that these IWs are described to first order by the Garrett-Munk IW spectrum. ${ }^{52}$ IW induced sound speed fluctuations are modeled in this study using the Garrett-Munk spectrum parameterized to fit shallow water observations. The mean square fractional sound-speed variability is given by

$$
\left\langle\mu(z)^{2}\right\rangle=\left\langle\delta c^{2}\right\rangle / c_{0}^{2}=\frac{\zeta_{0}^{2}}{c_{0}^{2}} \frac{n_{0}}{n_{B}(z)}\left(\frac{d c}{d z}\right)_{\mathrm{p}}^{2},
$$

where $c$ is the sound speed in meters per second $(\mathrm{m} / \mathrm{s}), \zeta_{0}$ is a reference displacement in meters, $c_{0}$ is a reference sound speed, and $n_{0}$ is a reference buoyancy frequency of $5.24 \times 10^{-3}$ radians per s (3 cycles per hour). Finally, the potential sound-speed gradient with depth, $(d c / d z)_{\mathrm{p}}$, is given in $\mathrm{s}^{-1}$ and is based on a potential temperature profile.

The Brunt-Vaisala frequency, $n_{B}$, is given by

$$
n_{B}^{2}(z)=-\frac{g}{\rho_{\mathrm{w}}(z)} \frac{\partial \sigma_{T}(z)}{\partial z},
$$

where $g$ is the acceleration of gravity in $\mathrm{m} / \mathrm{s}^{2}, \rho_{\mathrm{w}}$ is the seawater density in $\mathrm{kg} / \mathrm{m}^{3}$, and $\sigma_{T}$ is the density anomaly with the pressure reduced to atmospheric pressure in $\mathrm{kg} / \mathrm{m}^{3}$.

\section{Implementation of sound-speed perturbations in the propagation model}

A representative SSP, obtained during an experiment in the Gulf of Maine in September of 2010, was fitted with a Munk canonical profile ${ }^{53}$ following the form: 


$$
c(z)=c_{\min }\left[1+\xi\left(\eta+e^{-\eta}-1\right)\right],
$$

where $\eta=2\left(z-z_{\text {axis }}\right) / h_{0}$ and $c_{\min }=1472 \mathrm{~m} / \mathrm{s}$ is the minimum sound speed located at $z_{\text {axis }}=68 \mathrm{~m}$. Empirical parameter values used to fit the observed profile are $\xi=2.88$ $\times 10^{-3}$ and $h_{0}=48 \mathrm{~m}$. The resulting profile (Fig. 1, left panel) is used as the unperturbed SSP for all simulations incorporating depth and range dependent SSPs described in this study. This particular profile is also used in another modeling study in which the methods in this paper are applied to scattering by fish in the Gulf of Maine. ${ }^{34}$

IW-induced perturbations to the analytic SSP were generated via the Colosi and Brown method ${ }^{54}$ using observed profiles of buoyancy frequency, $n_{B}(z)$, and potential soundspeed gradient derived directly from the observed profile (Fig. 2). Parameters used to model the IWs were: The number of IW modes, $j_{\max }=15$, the mode bandwidth parameter, $j^{*}=1$, and the reference IW displacement, $\zeta_{0}=6$. These parameters were selected to reasonably represent a shallowwater IW field with a range-averaged, root-mean-squared sound-speed perturbation, $\delta c_{\mathrm{rms}}$, of approximately 2 to $5 \mathrm{~m} / \mathrm{s}$ in the main thermocline (Fig. 2). ${ }^{51}$ Finally, the sound-speed perturbations, $\delta c(r)$, as a function of range, $r$, were added to the analytic SSP in Eq. (15) and incorporated every $10 \mathrm{~m}$ in the propagation algorithm (Fig. 3). In the IW simulation a total of $2^{17}$ horizontal wavenumbers were used with a maximum wavenumber of $1 / 50 \mathrm{~m}$. These choices allow adequate sampling of the spectrum as well as providing smooth sound speed fluctuations in range. ${ }^{55}$

\section{Phasor summation method for generating echo PDFs}

The distribution of complex echo amplitudes for $N$ scatterers with arbitrary scattering responses randomly located in a directional beam can be determined using a Monte Carlo simulation in which the BP values, scattering amplitude, and phase are treated as random variables. The echo magnitude PDF, $\mathrm{p}_{E_{r}}(x)$, is determined from the
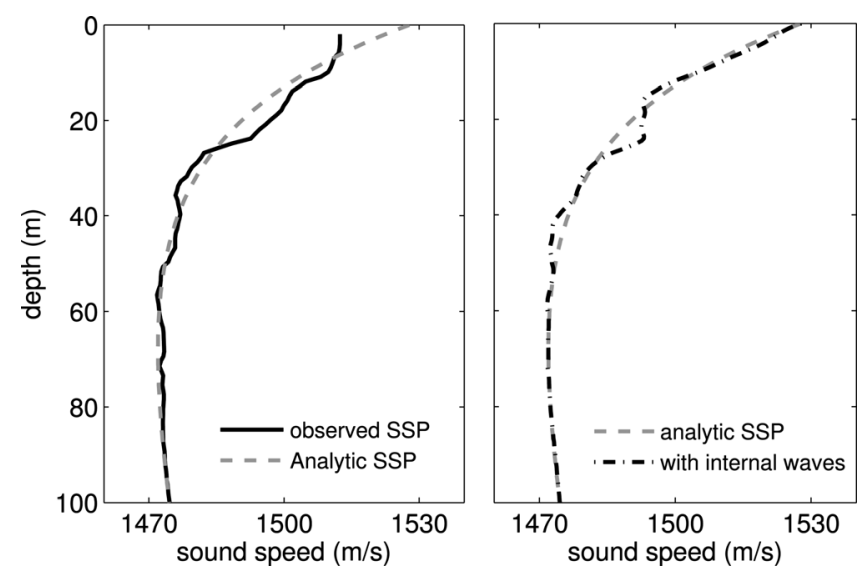

FIG. 1. Munk SSP fit to observed data, with and without IWs. Left panel shows a shallow water SSP measured in the Gulf of Maine $\left(42.0^{\circ} \mathrm{N}\right.$, $68.1^{\circ} \mathrm{W}$ ) in September of 2010 truncated at $100 \mathrm{~m}$ depth (solid line) and a canonical Munk SSP fit to the measured data (gray dashed line in both panels). Right panel shows a single realization of the same analytic Munk profile perturbed by diffuse IWs (black dotted-dashed line) as predicted by an empirically validated, shallow water IW model (Ref. 51).

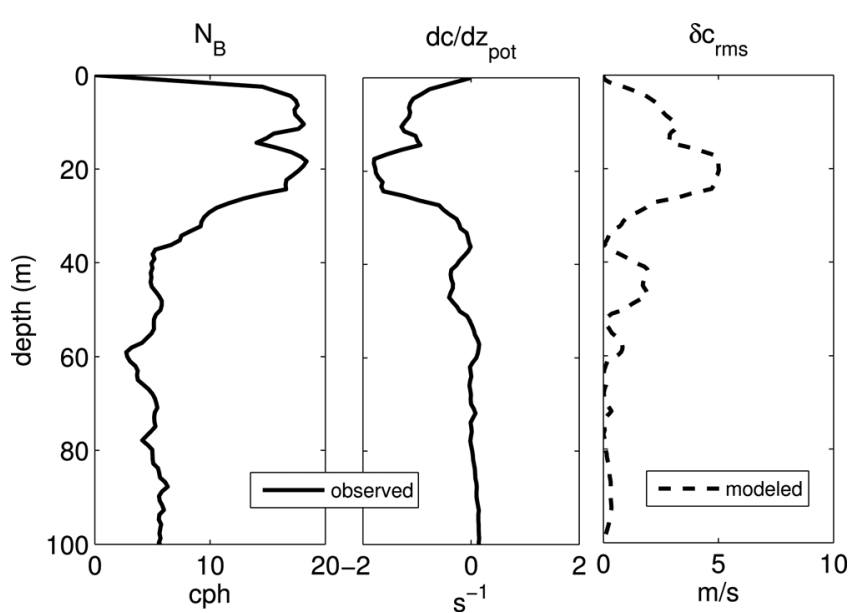

FIG. 2. Observed water column profiles from 2010 Gulf of Maine cruise. Buoyancy frequency and the derivative of potential sound speed with depth (left and center panel, respectively). Modeled range-averaged, rms soundspeed perturbation, $\delta c_{\mathrm{rms}}=c_{0} \sqrt{\left\langle\mu^{2}\right\rangle}$, using a reference sound speed, $c_{0}$, of $1472 \mathrm{~m} / \mathrm{s}$ (right panel).

normalized histogram of the echo envelopes, $E_{r}=\left|P_{r}\right|$. This numerical method is mathematically equivalent to solving Eq. (3) of Ref. 27, which analytically describes the characteristic function associated with the echo envelope distribution. Each realization of the simulation entails summing $N$ random phasors representing the sum of the complex scattered acoustic field with wavenumber, $k$, from $N$ scatterers as measured at a receiver

$$
P_{r}=\sum_{n=1}^{N} \frac{\Upsilon_{n}}{2\left|\vec{R}_{n}\right|} B_{n}^{\mathrm{S}}\left(\Theta_{\mathrm{az}}, \Theta_{\mathrm{el}}\right) B_{n}^{\mathrm{R}}\left(\Theta_{\mathrm{az}}, \Theta_{\mathrm{el}}\right) e^{2 \mathrm{i} k\left|\vec{R}_{n}\right|},
$$

where $B_{n}^{\mathrm{S}}$ and $B_{n}^{\mathrm{R}}$ represent the source and receiver BP weighting, respectively, as a function of the random azimuth, $\Theta_{\mathrm{az}}$, and DE, $\Theta_{\mathrm{el}}$, within an arbitrary beam. Points representing individual scatterers or aggregations of scatterers are positioned randomly with a uniform density ${ }^{56}$ at $\vec{R}_{n}$ on a spherical shell of thickness $\lambda / 2$, where $\lambda$ is the acoustic wavelength.

The stochastic term $\Upsilon_{n}$ is the combined complex waveguide response and complex scattering amplitude for the $n$th scatterer. In general, $\Upsilon_{n}$ must be determined numerically; however, two cases are presented in this study in which $\Upsilon_{n}$ is given in analytical form: (1) The case of a single, delta-

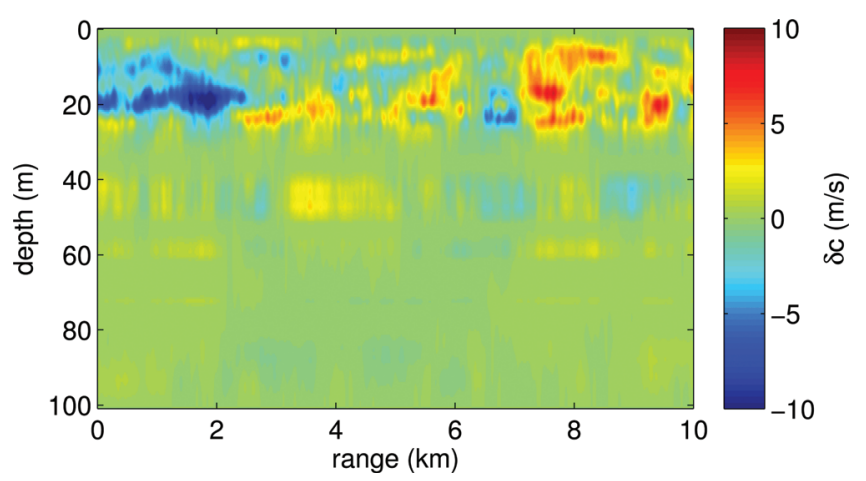

FIG. 3. A simulated realization of sound-speed perturbations due to diffuse, broadband IWs in shallow water using an empirically validated model (Ref. 51). 
function scatterer in a saturated forward field, where $\Upsilon_{n}$ is the square of a complex Gaussian random variable following Eq. (5), and (2) the case of an extended Rayleigh scatterer in a saturated forward field, where the term $\Upsilon_{n}$ is a zero-mean complex Gaussian random variable (with a Rayleigh envelope) as described in Sec. IIE. In addition to this stochastic term, waveguide effects are approximated, as described in Sec. II D, by constraining the range of $\Theta_{\mathrm{el}}$ narrowly about the horizontal.

\section{Statistical methods}

PDFs were calculated using a Monte Carlo method for two distinct cases: (a) The one-way propagation case which considers envelopes (i.e., the magnitude) of the pressure field at a fixed range and random depth and (b) the two-way propagation case which considers the envelopes of the echoes from one or more scatterers located at a fixed range and randomly located in depth and azimuth. Details of statistical methods used are given in Appendix A.

\section{E. Validation of models in direct path geometry with beam pattern effects}

The Chu-Stanton theory ${ }^{27}$ was used to validate both the two-way PE model and the numerical PS solution, Eq. (16). A direct path (i.e., no boundary effects) scenario was used for validation with $N$ scatterers located within the main lobe of a directional beam where $N=1,2,3,10$. Given the wide-angle limitation of the PE model (i.e., the model cannot accurately predict acoustic propagation at very wide angles of $\mathrm{DE})$, limiting the beam to the main lobe provides a way to benchmark the model. In this case the main lobe is defined by the polar angle at which the main lobe decreases to the value of the highest side lobe $\left(\theta_{\mathrm{SL}}\right)$. Since the Chu-Stanton theory applies to a sonar ensonifying scatterers in a direct path geometry, the parameters for the two-way PE model were selected to ensure boundary reflected echoes did not significantly interfere with the direct path signal (Table I).

Results of these calculations showed excellent agreement between all three models (Fig. 4). Due to the computationally intensive nature of the PE calculations, many fewer Monte Carlo realizations, $N_{\mathrm{MC}}$, were used $\left(N_{\mathrm{MC}, \mathrm{PE}}=1200\right)$ than in the PS solution $\left(N_{\mathrm{MC}, \mathrm{PS}}=5 \times 10^{5}\right)$ resulting in a scatter of the PE results that is greater than that of the PS. However, the results of the PE simulation still accurately predict the increased probability in the high amplitude portion of the PDF over a Rayleigh distribution (i.e., the heavy tail of the PDF) in all cases.

\section{SIMULATIONS OF ECHOES FROM A SINGLE SCATTERER IN A DETERMINISTIC WAVEGUIDE}

Before proceeding to studies of more realistic scattering scenarios, simple cases of increasing complexity involving a deterministic waveguide are first presented. Four sets of simulations were carried out using the numerical models described in Sec. III. The first case, a single scatterer with a constant, or delta function, scattering response (termed here a delta-function scatterer) ensonified by a point source in a
TABLE I. Model parameters simulating direct path geometry.

\begin{tabular}{|c|c|}
\hline Parameter & Value \\
\hline \multicolumn{2}{|l|}{ Sonar system parameters } \\
\hline Source type & circular piston $^{\mathrm{a}}(k a=37.06)$ \\
\hline Source depth (m) & 1500 \\
\hline Frequency $(\mathrm{kHz})$ & 3 \\
\hline Receiver type & circular piston $^{\mathrm{a}}(k a=37.06)$ \\
\hline Receiver depth (m) & 1500 \\
\hline \multicolumn{2}{|l|}{ Scatterer parameters } \\
\hline range from sonar $(\mathrm{m})$ & $1000 \pm \lambda / 2$ \\
\hline Location within beam & limited to range $\left[0, \theta_{\mathrm{SL}}\right]$ \\
\hline Scattering response & Rayleigh PDF \\
\hline \multicolumn{2}{|l|}{ Environmental parameters } \\
\hline $\mathrm{SSP}(\mathrm{m} / \mathrm{s})$ & 1500 (constant) \\
\hline Water depth (m) & 3000 \\
\hline Bottom density $\left(\mathrm{kg} / \mathrm{m}^{3}\right)$ & $1000^{\mathrm{b}}$ \\
\hline Bottom speed $(\mathrm{m} / \mathrm{s})$ & $1500^{\mathrm{b}}$ \\
\hline Bottom attenuation $(\mathrm{dB} / \lambda)$ & $200^{\mathrm{b}}$ \\
\hline \multicolumn{2}{|l|}{ PE model parameters } \\
\hline range step $(\mathrm{m})$ & 5 \\
\hline Depth step (m) & 0.05 \\
\hline \# of Padé terms & 4 \\
\hline
\end{tabular}

${ }^{\text {a }}$ Source and receiver are co-located (monostatic).

${ }^{\mathrm{b}}$ Parameters simulate a transparent, highly absorbent bottom.

Pekeris waveguide, provides a baseline for comparison with models of greater complexity. Each subsequent case presented is then compared with this result to understand how varying environmental conditions and sonar specifications cause the echo statistics to deviate from this simple case. These additional factors are: A depth-dependent SSP, a directional sonar system, and an extended scatterer with a variable scattering response. In the present cases, statistics
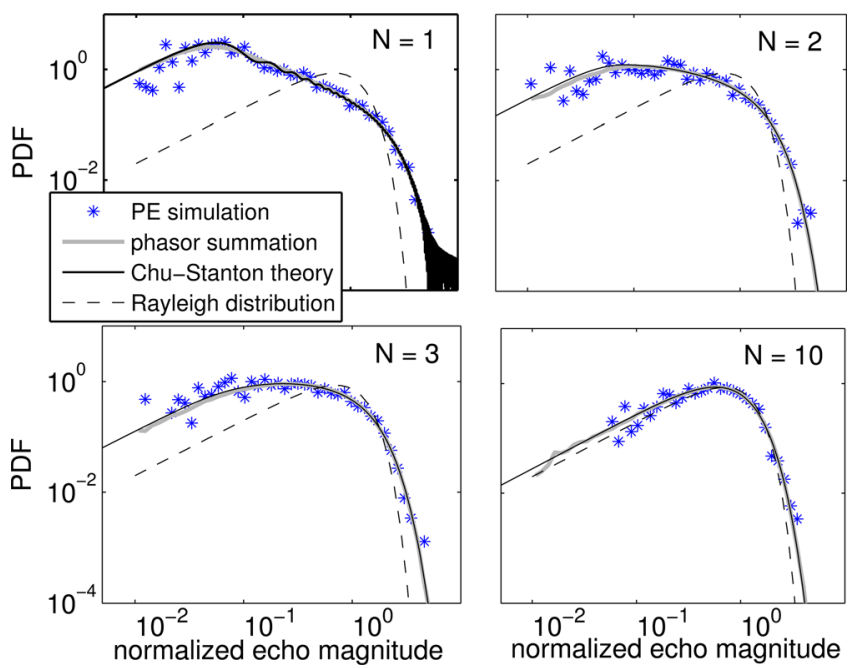

FIG. 4. (Color online) Predicted echo PDFs of various numbers of scatterers, each with a Rayleigh distributed scattering amplitude, randomly located in the main lobe of a directional sonar beam in a direct path geometry. The sonar is a monostatic, circular piston with a $3 \mathrm{kHz}$ source and $5^{\circ}$ beam widths (both transmit and receive). Asterisks are predictions using the twoway PE simulation at a range of $1 \mathrm{~km}$, gray solid line is numerical PS which incorporates BP effects, black solid line is theoretical curve (Ref. 27), and the dashed black line is a Rayleigh distribution. The PS and Chu-Stanton theoretical curves are virtually identical masking much of the black solid line. The number of scatterers, $N$, is given in the top right of each panel. 
are compiled by randomly positioning the scatterer in depth at a fixed range.

\section{A. Delta-function scatterer ensonified by an omnidirectional source in a constant sound-speed environment}

In this section we consider the echo statistics associated with a $3 \mathrm{kHz}$ omnidirectional source ensonifying a single delta-function scatterer randomly located in the water column at a fixed range. In this case the water depth is $100 \mathrm{~m}$ and has a constant sound speed, and the source is located at $50 \mathrm{~m}$ depth (see Table II, case A for key model parameters). Though the focus of this study is the PDF of the envelope of the echo from a randomly located scatterer, the forward field should first be considered. This outgoing acoustic field is characterized by a highly structured interference pattern caused by multipath due to multiple boundary reflections [Fig. 5(a)].

At $1 \mathrm{~km}$ range there is sufficient multipath interference to yield a Rayleigh PDF for the one-way pressure field (Fig. 6; top panel). A Lilliefors test verifies these results: The uncorrelated real and imaginary components of the pressure distribution are compared, individually, with a normal distribution (correlation coefficient, $\rho_{\text {cor }}=5.7 \times 10^{-3}$; $p$-values: $p_{\text {real }} / p_{\text {imag. }}=2.23 \times 10^{-1} / 8.90 \times 10^{-2}$ )—see Appendix A for details of statistical methods used in this study.

Once the scattered signal travels back to the source, the envelopes of the received echoes are exponentially distributed $(p=0.35)$ as described by the theory in Sec. II C (Fig. 6; bottom panel).

\section{B. Effects of a depth-dependent sound-speed profile}

A significant amount of environmental realism can be added to the model by incorporating a realistic SSP (see Table II, case C). With a depth-dependent SSP, some of the energy is trapped in the sound channel, a phenomenon that is
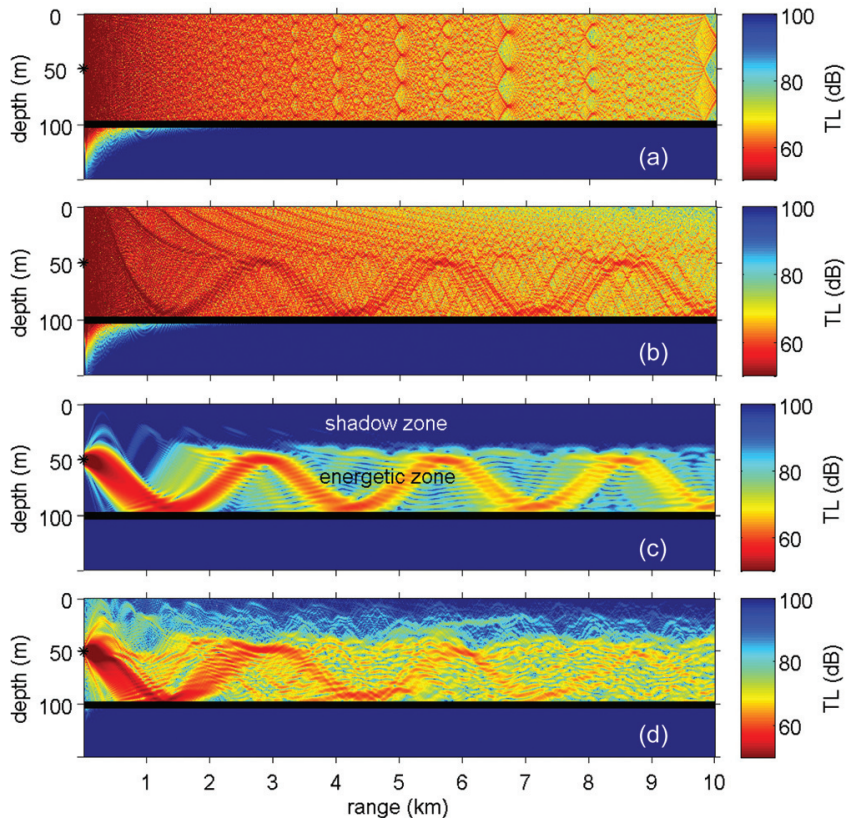

FIG. 5. Predicted transmission loss from a $3 \mathrm{kHz}$ source located at $50 \mathrm{~m}$ depth in an oceanic waveguide with varying levels of complexity. (a) Omnidirectional source, constant SSP; (b) omni-directional source, canonical Munk SSP; (c) directional source, Munk SSP; (d) directional source, Munk SSP with IW perturbations. In (c) and (d) the directional source is a circular piston $(\mathrm{ka}=37.06)$ with a narrow beam $\left(5^{\circ}-3 \mathrm{~dB}\right.$ beam width $)$.

weakly detectable in a transmission loss plot [Fig. 5(b)]. The statistics at two ranges are presented in this case- 1 and $3.5 \mathrm{~km}$ (Fig. 7). The forward propagated pressure field, when sampled at random depths, approaches a Rayleigh distribution. Unlike the constant sound-speed case, the one-way field is not Rayleigh distributed by $1 \mathrm{~km}$ but does reach a Rayleigh distribution by $3.5 \mathrm{~km}\left(\rho_{\text {cor }}=-2.47 \times 10^{-2}\right.$; $\left.p_{\text {real }} / p_{\text {imag. }}=0.83 / 0.36\right)$. In the back-propagated case, the

TABLE II. Key modeling parameters used in numerical simulations. Parameters which were constant throughout all simulations include the source frequency: $3 \mathrm{kHz}$; bottom characteristics: density $=2000 \mathrm{~kg} / \mathrm{m}^{3}$, sound speed $=1600 \mathrm{~m} / \mathrm{s}$, and attenuation $=0.5 \mathrm{~dB} / \mathrm{wavelength}$; and $\mathrm{PE}$ modeling parameters: range step $=1 \mathrm{~m}$, depth step $=0.025 \mathrm{~m}$, and number of Padé terms $=8$.

\begin{tabular}{|c|c|c|c|c|c|c|c|c|}
\hline Case & A & $\mathrm{B}$ & $\mathrm{C}$ & $\mathrm{D}$ & $\mathrm{E}$ & $\mathrm{F}$ & G & $\mathrm{H}$ \\
\hline \multicolumn{9}{|c|}{ Source/receiver parameters } \\
\hline Source type ${ }^{a}$ & omni & omni & omni & beam & beam & beam & beam & beam \\
\hline Source depth & $50 \mathrm{~m}$ & $50 \mathrm{~m}$ & $50 \mathrm{~m}$ & $50 \mathrm{~m}$ & $50 \mathrm{~m}$ & $10 \mathrm{~m}$ & $10 \mathrm{~m}$ & $10 \mathrm{~m}$ \\
\hline Receiver type $^{\mathrm{b}}$ & omni & omni & omni & omni & omni & omni & omni & omni \\
\hline Receiver depth & $50 \mathrm{~m}$ & $50 \mathrm{~m}$ & $50 \mathrm{~m}$ & $50 \mathrm{~m}$ & $50 \mathrm{~m}$ & $10 \mathrm{~m}$ & $10 \mathrm{~m}$ & $10 \mathrm{~m}$ \\
\hline \multicolumn{9}{|c|}{ Scatterer parameters } \\
\hline Location $^{\mathrm{c}}$ & vertical plane & vertical plane & vertical plane & on axis & on axis & on axis & full-beam & full-beam \\
\hline Response $^{\mathrm{d}}$ & delta & Rayleigh & delta & delta & delta & delta & delta & Rayleigh \\
\hline \multicolumn{9}{|c|}{ Environmental parameters } \\
\hline $\mathrm{SSP}^{\mathrm{e}}$ & constant & constant & Munk fit & Munk fit & Munk fit & Munk fit & Munk fit & Munk fit \\
\hline $\mathrm{IWs}^{\mathrm{f}}$ & no & no & no & no & yes & yes & yes & yes \\
\hline
\end{tabular}

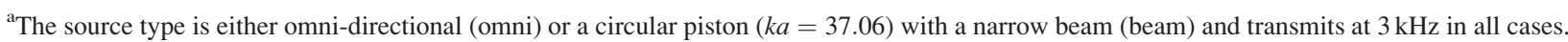

${ }^{\mathrm{b}}$ The receiver type is omni-directional (omni) in all cases.

${ }^{\mathrm{c}}$ The scatterer is either randomly located in DE at a fixed range from the source (vertical plane), randomly located in DE and azimuthally on axis of the main lobe (on axis), or randomly located in DE and azimuth (full beam).

${ }^{\mathrm{d}}$ The scattering response denotes a scattering magnitude that is either fixed at unity (delta function) or Rayleigh distributed (Rayleigh).

${ }^{\mathrm{e}}$ The water column sound speed is either a constant $1500 \mathrm{~m} / \mathrm{s}$ (constant) or an analytical fit of a Munk SSP to an observed shallow water profile (Munk fit).

${ }^{\mathrm{f}} \mathrm{A}$ yes for IWs indicate that the SSP is perturbed every $10 \mathrm{~m}$ with a shallow water IW model. 

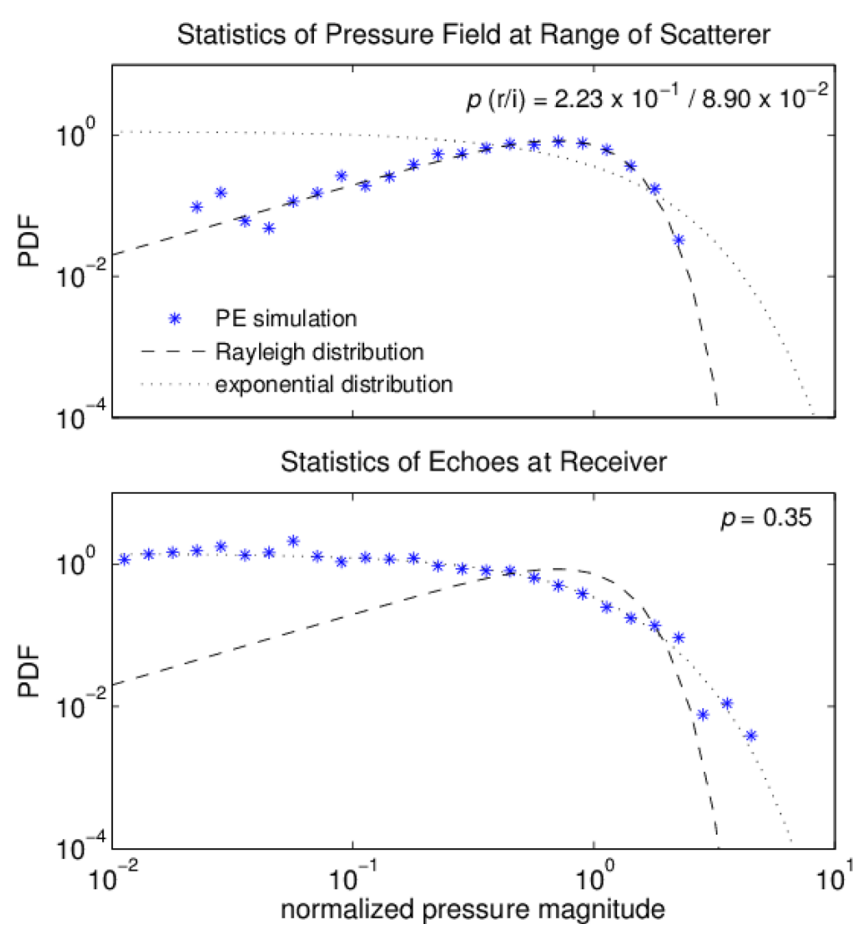

FIG. 6. (Color online) Predicted PDFs associated with a $3 \mathrm{kHz}$ omnidirectional source at a depth of $50 \mathrm{~m}$ ensonifying a single delta-function scatterer randomly located in depth at a range of $1000 \mathrm{~m}$ in a waveguide with a constant SSP (case A, Table II). Top panel is the PDF of the magnitude of the pressure field at the scatterers random location and the bottom panel is the PDF of the echo envelope at the receiver. Lilliefors test $p$-values for comparison with the Rayleigh distribution (top panel) and the exponential distribution (bottom panel) are given in the top right corner of each panel.

PDF of the echoes at the receiver is approaching an exponential as can be seen by the small Kullback-Leibler distances, $d_{\mathrm{KL}} \quad\left(d_{\mathrm{KL}}=2.19 \times 10^{-2}\right.$ at $1 \mathrm{~km} ; d_{\mathrm{KL}}=2.95 \times 10^{-2}$ at $3.5 \mathrm{~km})$ compared with that at $500 \mathrm{~m}\left(d_{\mathrm{KL}}=4.75 \times 10^{-2}-\right.$ not shown). However, the PDF does not pass a statistical test for an exponential distribution at either range modeled.

\section{Effects of an elevation-dependent beam pattern}

A key parameter affecting echo statistics is the sonar $\mathrm{BP}^{46}$ A directional source is modeled to emulate a circular piston transducer with a wavenumber-radius product, $\mathrm{ka}$, of 37.06 (see Table II, case D). This source produces an axisymmetric composite (two-way) BP with a narrow $5^{\circ}-3 \mathrm{~dB}$ beam width at $3 \mathrm{kHz}$. In order to identify effects corresponding only to the directionality of the beam in the vertical plane, the scatterer in this simulation was randomly located in depth, while remaining azimuthally on-axis. Thus, these simulations are akin to a vertical line array with a BP that is dependent upon DE, but constant in azimuth.

The most noticeable effect of the addition of the directional source is to produce a distinct shadow zone in the forward pressure field as can be seen in the transmission loss plot [Fig. 5(c)]. Simulations were conducted with a single scatterer randomly located in depth within two separate regions: (1) The shadow zone $(0-40 \mathrm{~m}$ depth) and (2) the energetic zone (40-100 $\mathrm{m}$ depth). At the ranges investigated up to $9.5 \mathrm{~km}$, only a weak range-dependence exists in the echo statistics distributions (Fig. 8). All distributions broadly
Statistics of Pressure Field at Range of Scatterer

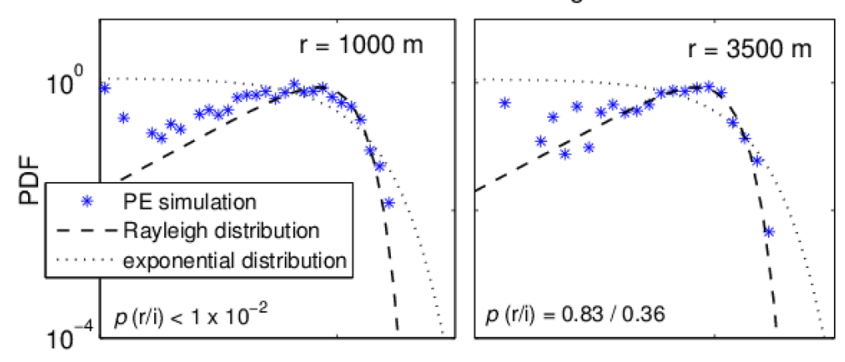

Statistics of Echoes at Receiver

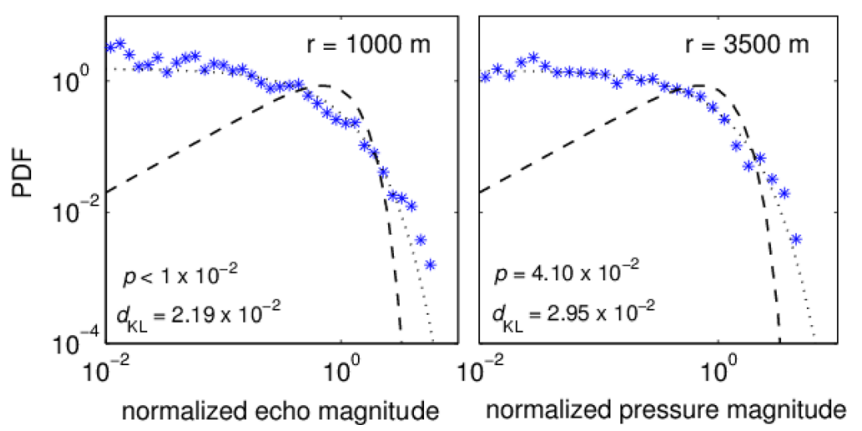

FIG. 7. (Color online) Predicted PDFs associated with a $3 \mathrm{kHz}$ omnidirectional source at a depth of $50 \mathrm{~m}$ ensonifying a single scatterer randomly located in depth at a range of $1000 \mathrm{~m}$ (left two panels) and $3500 \mathrm{~m}$ (right two panels) in a waveguide with a canonical Munk SSP (case C, Table II). Top two panels give the statistics of the magnitude of the pressure field at the scatterers random location, while the bottom two panels give the statistics of the echo envelope at the receiver. Lilliefors test $p$-values for comparison with the Rayleigh distribution (top panels) and the exponential distribution (bottom panel) are given in the bottom left corner of each panel. KullbackLeibler distances comparing model predictions with the exponential distribution, $d_{\mathrm{KL}}$, are given in the bottom left corner of the bottom panels.

resemble the exponential rather than the Rayleigh, but with some notable deviations.

For scatterers positioned solely in the shadow zone, the tails of the distributions are strongly non-Rayleigh and nonexponential. However, the mean of the echo amplitudes is very low in this scattering geometry so the PDFs here have little practical value. In the energetic zone, the pressure field at the scatterer does not reach a Rayleigh distribution at any of the ranges simulated up to $9.5 \mathrm{~km}\left(4.61 \times 10^{-2} \leq d_{\mathrm{KL}}\right.$ $\leq 1.48 \times 10^{-1}$-not shown). At the receiver, the echo PDFs are initially (at a range to the scatterer of $500 \mathrm{~m}$ ) strongly non-exponential $\left(d_{\mathrm{KL}}=7.17 \times 10^{-2}\right)$, but become more exponential-like at ranges from 3.5 to $9.5 \mathrm{~km}\left(2.65 \times 10^{-2}\right.$ $\leq d_{\mathrm{KL}} \leq 5.73 \times 10^{-2}$ ). In all cases the distribution failed a Lilliefors test for goodness of fit with an exponential distribution. These results suggest that, at least at these short ranges where much of the sound energy is coherently refracted into the sound channel, the limited deterministic multipath may not be sufficient to induce an exponential distribution as seen in case A with the omnidirectional source.

\section{Scatterer of varying spatial extent with a variable scattering response (Rayleigh scatterer)}

The formulation in Sec. IIE is validated through simulations of spherical Rayleigh scatterers of varying radii located in a complex-Gaussian distributed acoustic pressure field 

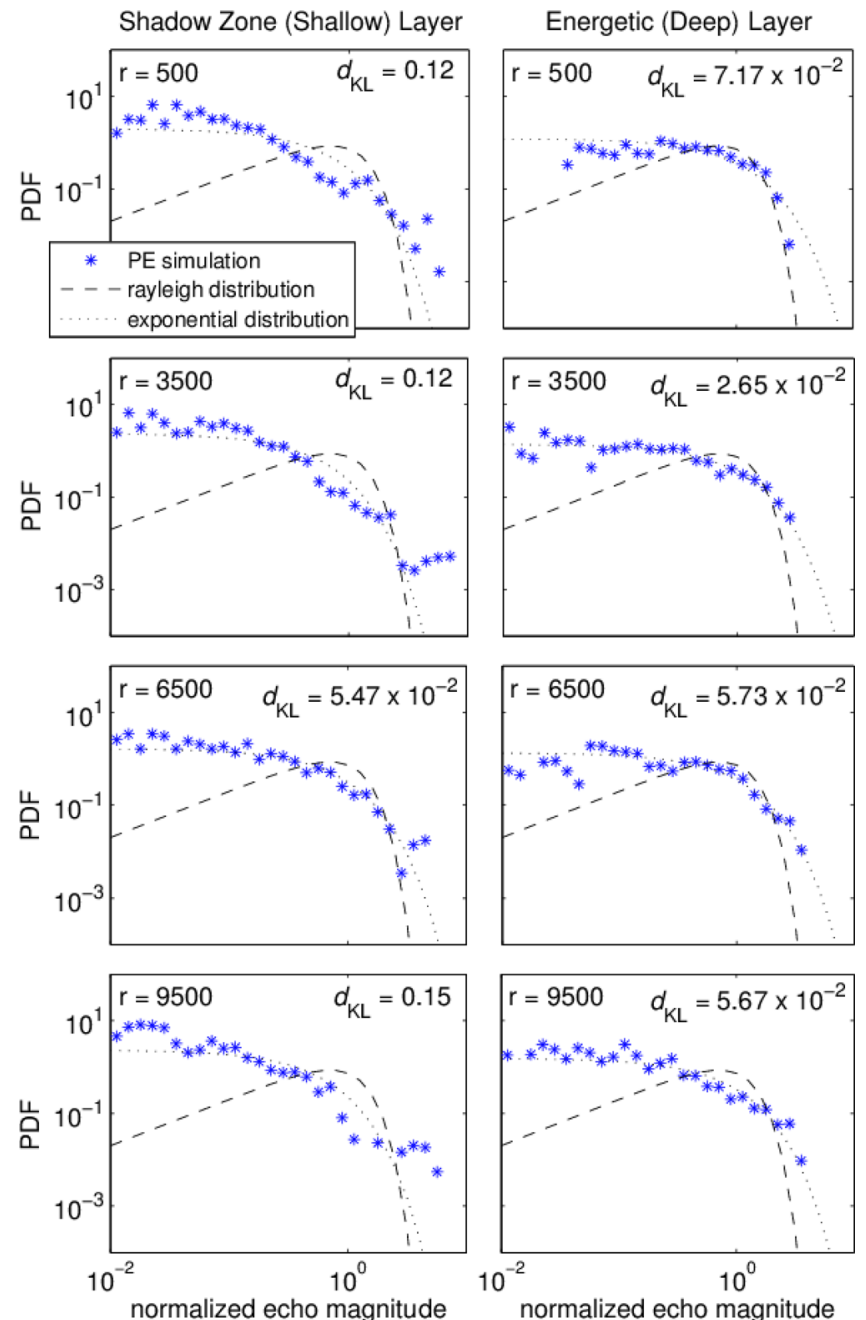

FIG. 8. (Color online) Predicted echo PDFs associated with a $3 \mathrm{kHz}$ directional source at a depth of $50 \mathrm{~m}$ ensonifying a single scatterer located azimuthally on axis and randomly in depth at various ranges in a waveguide with a canonical Munk SSP (case D, Table II). The panels on the left give the statistics of the shadow zone ( 0 to $40 \mathrm{~m}$ ), and the panels on the right give the statistics of the energetic zone ( 40 to $100 \mathrm{~m}$ ). The range of the scatterer, $r$, is given in the top left of each panel. The directional source is a circular piston $(k a=37.06)$ with a narrow beam $\left(5^{\circ}-3 \mathrm{~dB}\right.$ beam width) and the scatterer response is a constant. Kullback-Leibler distances comparing model predictions with the exponential distribution, $d_{\mathrm{KL}}$, are given in the top right corner of each panel.

(see Table II, case B). Each Rayleigh scatterer is modeled using 10 point scatterers uniformly distributed within a sphere of a given radius, $r_{V}$. A Monte Carlo simulation with an omni-directional source and ten scatterers in a direct path geometry shows that ten scatterers is sufficient to generate a Rayleigh PDF ( $p_{\text {real }} / p_{\text {imag. }}=0.86 / 0.88$-figure not shown). To simulate Rayleigh scatterers of varying size, $r_{V}$ is varied from $0.125 \mathrm{~m}$ (a two-way travel distance across the scatterer of 1 wavelength) to $4.0 \mathrm{~m}$ (two-way travel of 32 wavelengths). Note the very small Rayleigh scatterers here, $r_{V}$ $\leq 0.125 \mathrm{~m}$, may not be representative of small, dense aggregations where other effects, purposefully ignored in this study, may be important (e.g., multiple scattering, attenuation-as discussed in Sec. I). However, all cases can be applied to individual scatterers with a ping-to-ping response that is Rayleigh distributed (e.g., a randomly rough, randomly oriented sphere).
Monte Carlo simulations were conducted comparing a Rayleigh point target (i.e., an infinitesimally small scatterer located at a single point with a magnitude drawn from a Rayleigh distribution for each random instance) with a Rayleigh scatterer of varying size (Fig. 9). All cases are compared with the exponential distribution which arises from a single delta-function scatterer in a complex-Gaussian distributed pressure field as shown in Sec. IV A. In the case of a very small radius, $r_{V}=0.125 \mathrm{~m}$, the echo envelope distribution is exponential-like with a slightly heavier tail very similar to the result of the Rayleigh point target. According to the theory, the distribution in this case approaches the product of an exponential and a Rayleigh random variable. As the radius increases the distribution begins to approach a Rayleigh distribution as predicted $\left(d_{\mathrm{KL}}=7.14 \times 10^{-2}\right.$ for $r=2.0 \mathrm{~m}$ and $1.35 \times 10^{-2}$ for $r=4.0 \mathrm{~m}$ ).

\section{SIMULATIONS OF ECHOES FROM SINGLE AND MULTIPLE SCATTERERS IN A RANDOM WAVEGUIDE}

Stochastic ocean factors that are known to cause acoustic variability are small scale bottom roughness, surface waves, and IWs. As a first attempt at simulating a realistic
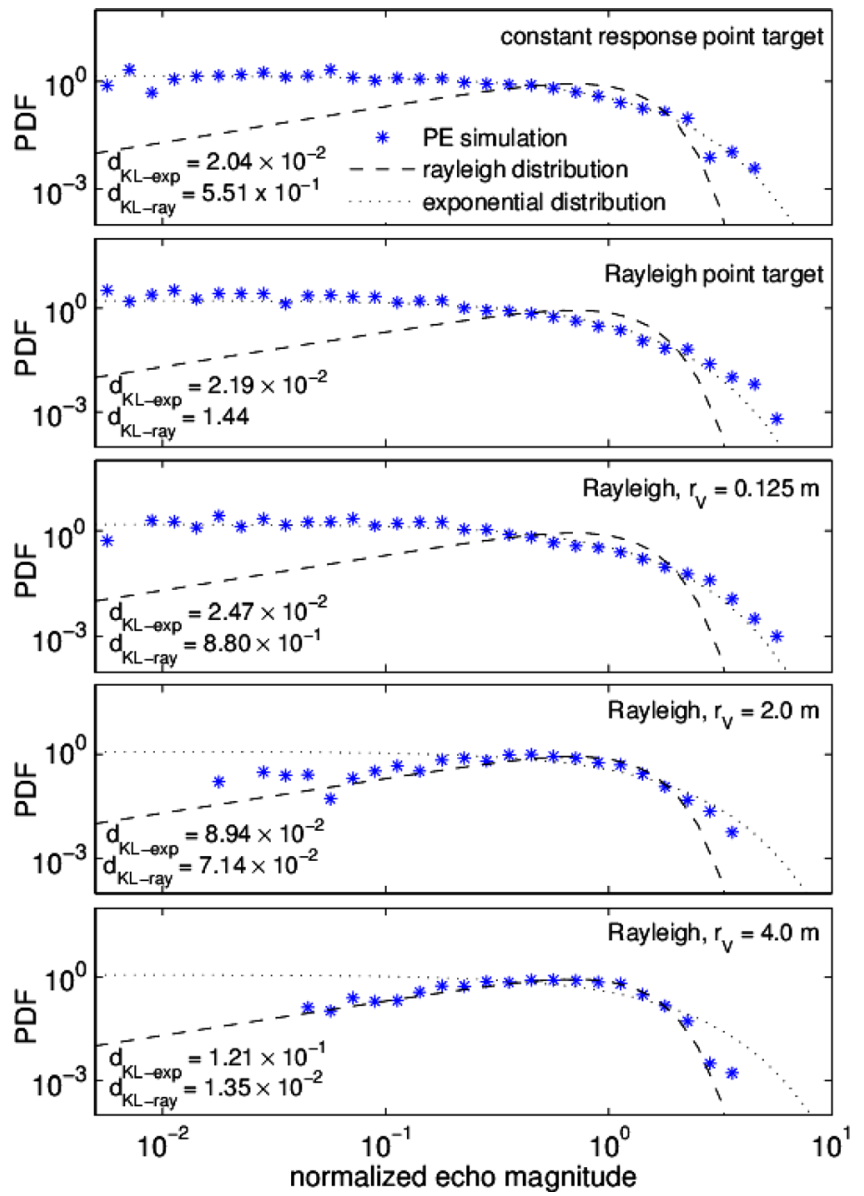

FIG. 9. (Color online) Predicted echo PDFs associated with a $3 \mathrm{kHz}$ omnidirectional source at a depth of $50 \mathrm{~m}$ ensonifying a single spherical Rayleigh scatterer of various radii, $r_{v}$. The scatterer is randomly located in depth at a range of $1000 \mathrm{~m}$ in a waveguide with a constant SSP (case B, Table II). The top panel is the echo PDF of a delta-function scatterer with the same parameters for comparison. Kullback-Leibler distances comparing model predictions with the exponential distribution, $d_{\mathrm{KL}-\exp }$, and the Rayleigh distribution, $d_{\mathrm{KL}-\text { ray }}$, are given in the bottom left corner of each panel. 
ocean environment and the corresponding effects on the echo statistics, random fields of IW-induced sound-speed perturbations are simulated (e.g., Fig. 1, right panel). IW fields are chosen because the simulation tools are well established ${ }^{54}$ and the acoustical effects of these waves have been well studied. ${ }^{23,24,57}$ Here we focus on the cases of both a single scatterer and multiple scatterers ensonified by a directional sonar beam.

Stochastic sound-speed structure is important in this context, as it leads to additional signal randomization (stochastic multipath) beyond that caused by randomizing the depth of the scatterer. Additionally, the sound-speed structure causes forward scattering into the shadow zone $^{58-60}$ as demonstrated [Figs. 5(c) and 5(d)]. In Fig. 5(d) the coherent beam illustrated in Fig. 5(c) fractures into many micropaths, increasing randomization and inducing interference. In addition, Fig. 5(d) shows an increased ensonification of the shadow zone relative to Fig. 5(c).

\section{A. Single scatterer in an elevation-dependent beam pattern}

In this simulation, echo envelope PDFs for a single scatterer in a directional BP were calculated from 3000 random realizations of the two-way PE model, each of which included a random realization of the IW field and a scatterer randomly located in depth (see Table II, case E). The results were again divided into two cases depending on the location of the scatterer: Either in the shadow zone $(\leq 40 \mathrm{~m})$ or in the energetic zone $(>40 \mathrm{~m})$. The effects of the stochastic component of the waveguide (Fig. 10) can be seen by comparing with the deterministic case (Fig. 8). For scatterers solely distributed in the shadow zone the tails of the echo distributions are highly elevated above both the Rayleigh and exponential distributions at all ranges from $500 \mathrm{~m}$ to $9.5 \mathrm{~km}$. The primary difference between this case and the deterministic case is that the tail is consistently elevated out to higher amplitudes in the stochastic case. This is most likely due to the strong leakage of energy into the shadow zone. In the energetic zone the envelope of the outgoing pressure field is Rayleigh or near-Rayleigh-distributed at ranges $\geq 3.5 \mathrm{~km}\left(d_{\mathrm{KL}} \leq 2.18\right.$ $\times 10^{-2}$-figures not shown). The effect of this is seen in the echo PDFs, where the tails match the exponential distribution at all ranges $\geq 3500 \mathrm{~m}$ (Fig. 10). Furthermore, the Kullback-Leibler distance decreases at each consecutive range step that was simulated reducing from $3.53 \times 10^{-2}$ at $500 \mathrm{~m}$ to $3.01 \times 10^{-2}$ at $9500 \mathrm{~m}$.

\section{B. Multiple scatterers in an elevation-dependent beam pattern}

Next, simulations were conducted to investigate the effects of first-order scattering by multiple scatterers. Here, "first-order" refers to the scattering of acoustic waves directly from the source without interference from other scatterers. The model setup in Sec. V A was applied with $N$ scatterers distributed randomly in the vertical plane that contains the main axis of the sonar and within the energetic zone (40 to $100 \mathrm{~m}$ ) at a range of $10 \mathrm{~km}$. The echo envelope PDFs were calculated for $N=1,2,3$, and 10 (Fig. 11). For $N=1$, the echo envelope PDF is exponential as before. However, it is evident that the
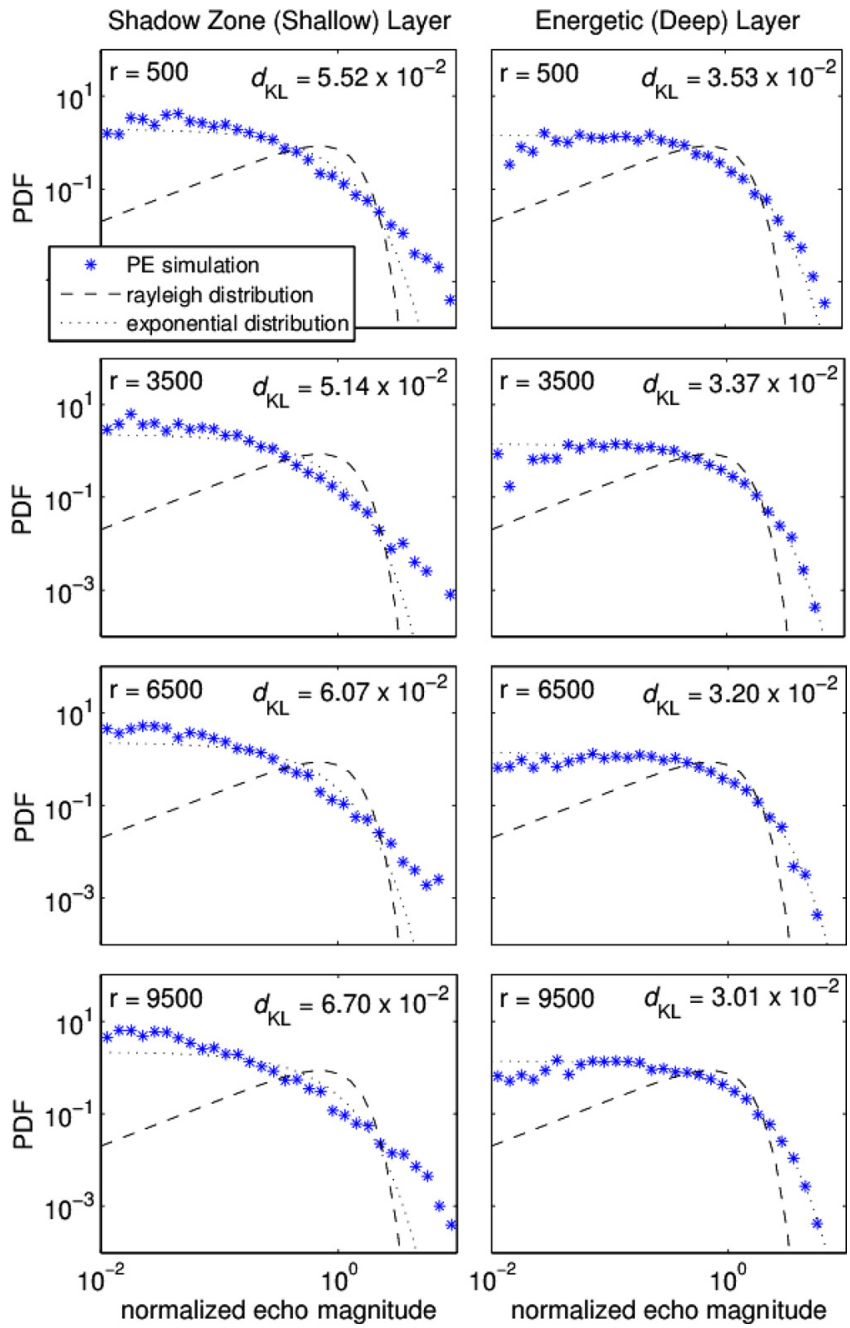

FIG. 10. (Color online) Predicted echo PDFs associated with a $3 \mathrm{kHz}$ directional source at a depth of $50 \mathrm{~m}$ ensonifying a single scatterer located azimuthally on axis and randomly in depth at various ranges in a random waveguide (case E, Table II). The panels on the left give the statistics of the shadow zone ( 0 to $40 \mathrm{~m}$ ), and the panels on the right give the statistics of the energetic zone (40 to $100 \mathrm{~m}$ ). The range of the scatterer, $r$, is given in the top left of each panel. The directional source is a circular piston $(k a=37.06)$ with a narrow beam $\left(5^{\circ}-3 \mathrm{~dB}\right.$ beam width) and the scatterer response is a constant. Kullback-Leibler distances comparing model predictions with the exponential distribution, $d_{\mathrm{KL}}$, are given in the top right corner of each panel.

PDF is trending toward a Rayleigh with increasing N. At this range with a saturated incident field at each scatterer, each scatterer produces an independent contribution to the echo, leading to a complex Gaussian distribution of echoes for large $N$ by the CLT (e.g., see Goodman). ${ }^{61}$

\section{SIMULATIONS OF ECHOES FROM SINGLE AND MULTIPLE SCATTERERS IN A THIN LAYER WITHIN A RANDOM WAVEGUIDE}

In order to address a more realistic scenario, simulations were conducted with a directional source located at $10 \mathrm{~m}$ depth and scatterers located randomly within a $10 \mathrm{~m}$ thick layer (simulating biologic scatterers which are often stratified in thin horizontal layers in the ocean). Of particular interest is the degree to which the echo magnitude PDFs are non-Rayleigh and how the BP and scattering response affect these statistics. Two cases, with $N$ scatterers randomly 


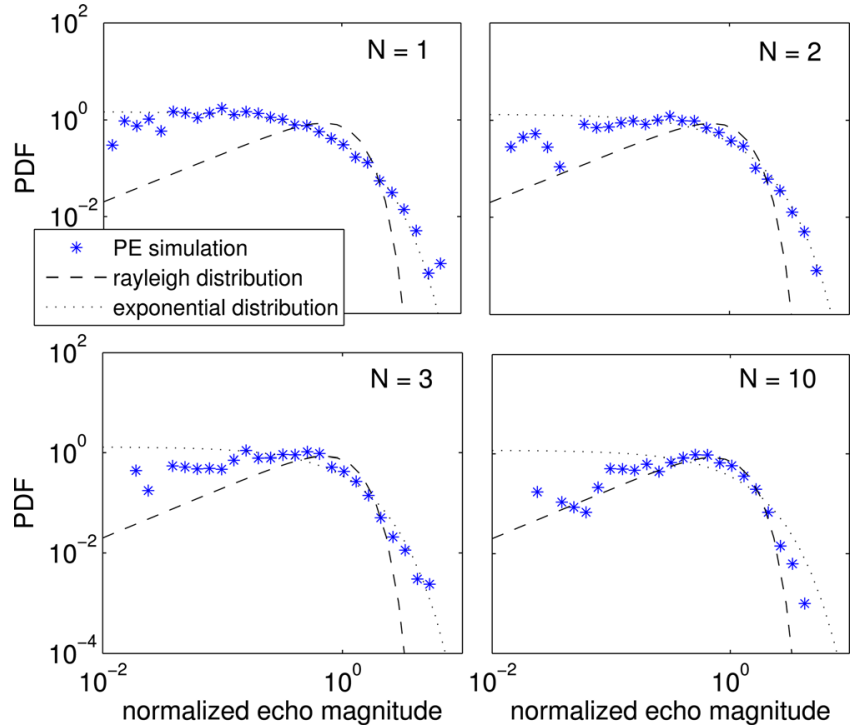

FIG. 11. (Color online) Predicted echo PDFs associated with a $3 \mathrm{kHz}$ directional source at a depth of $50 \mathrm{~m}$ ensonifying various numbers of scatterers in a random waveguide. The scatterers are located azimuthally on axis and randomly in depth in the energetic zone (40-100 m depth) at a range of $10 \mathrm{~km}$ (case E, Table II). The directional source is a circular piston $(k a=37.06)$ with a narrow beam $\left(5^{\circ}-3 \mathrm{~dB}\right.$ beam width). The number of scatterers, $N$, is given in the top right of each panel. The scatterers are at a range of $10 \mathrm{~km}$ in all cases and have a constant scattering response.

located in depth within a thin layer, were investigated: A shallow layer in the thermocline from 10 to $20 \mathrm{~m}$ depth and a deep layer near the bottom from 85 to $95 \mathrm{~m}$ depth (see horizontal white lines on Fig. 12). The number of scatterers was also varied with $N=1,2,5$, and 10 .

Calculations of unperturbed and perturbed transmission loss for this more realistic directional source depth are
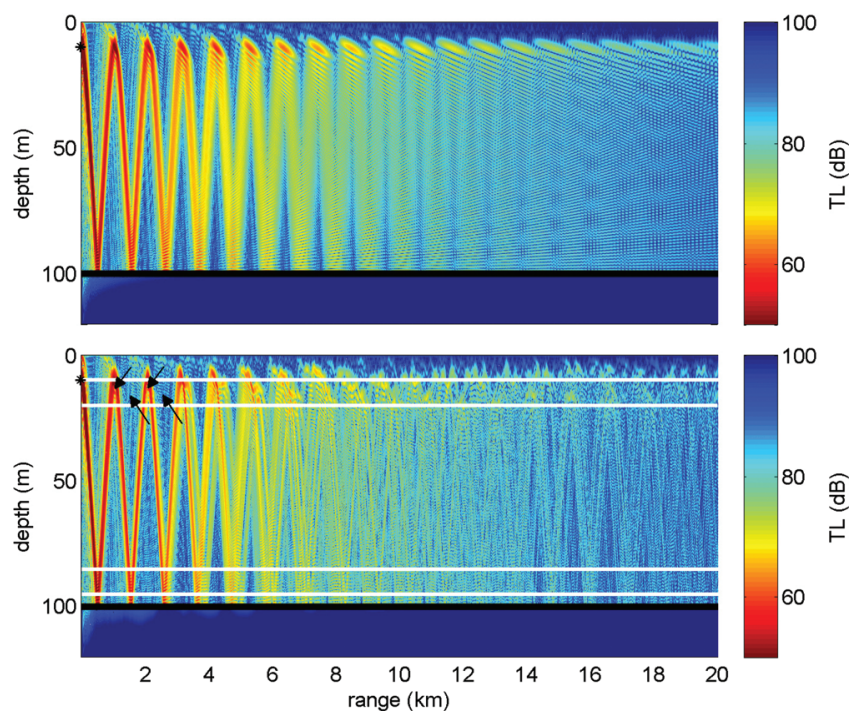

FIG. 12. Predicted transmission loss from a $3 \mathrm{kHz}$ directional source located at $10 \mathrm{~m}$ depth. The top panel is a range independent waveguide with a Munk SSP, while the bottom panel is a range-dependent waveguide with a Munk SSP perturbed by diffuse IWs. The directional source is a circular piston $(k a=37.06)$ with a narrow beam $\left(5^{\circ}-3 \mathrm{~dB}\right.$ beam width). Pairs of white lines in the lower panel represent the boundaries of two layers used in the analysis: A shallow layer (10-20 m) and a deep layer (85-95 m). Arrows in the lower panel depict energetic zones (top two arrows) and shadow zones (bottom two arrows) within the layer analyzed. illustrated (Fig. 12). It is evident that the shadow zone in the upper ocean is significantly reduced relative to the case shown in Fig. 5(c). However, the acoustic beam is much more collimated, giving rise to shadow zones horizontallyspaced between the paths of the acoustic beam. The two layers in which the scatterers are located in this analysis are each below the shallow shadow zone. Two range-dependent effects are observed. First, a periodic effect is observed in the echo statistics that corresponds to the scatterer location passing in and out of the energetic beam with range. Second, a trend toward saturation can be seen with an increase in range.

\section{A. Single scatterer in an elevation-dependent BP}

In order to quantify the range dependence of the echo characteristics, the SI was chosen as a good measure of the degree to which echo statistics approach a saturated state. The SI is a particularly useful measure in this context, as it is most sensitive to the high amplitude portion of the intensity PDF of a signal. Given a known amplitude distribution, SI can be determined using a multipath interference model, ${ }^{41}$ as detailed in Appendix B. For example, in the case in which a single scatterer in a random waveguide is ensonified by a directional source the signal reaches saturation for both the forward problem (one-way propagation) and the backscattered field at the receiver corresponding to $\mathrm{SI}=1$ and 5 , respectively (Fig. 13).

Two scattering layers in a $100 \mathrm{~m}$ deep waveguide (10-20 and $85-95 \mathrm{~m}$ ), ensonified by a shallow directional source, are considered separately (see Table II, case F). Two

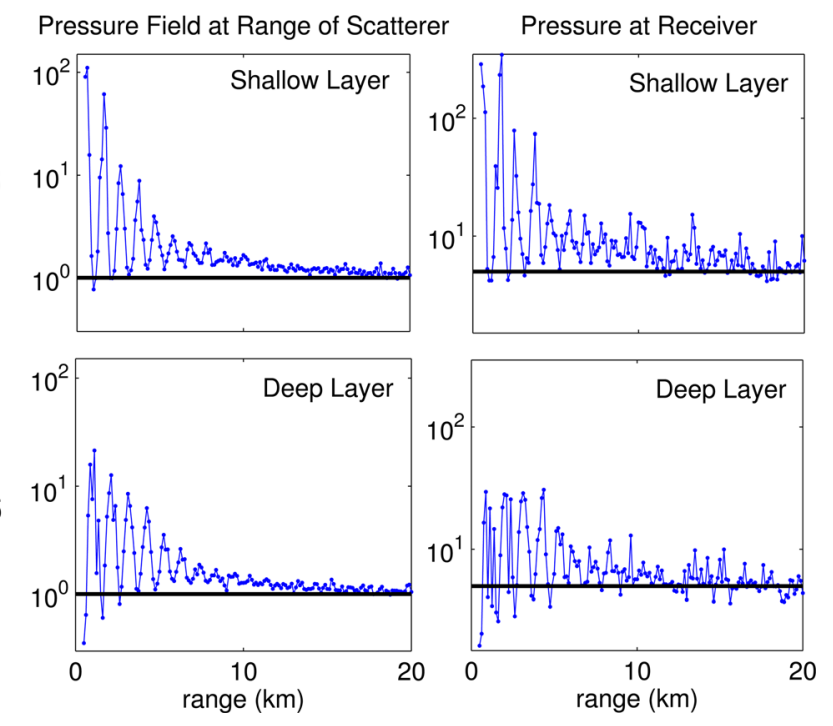

FIG. 13. (Color online) Predicted normalized intensity variance (SI) associated with a $3 \mathrm{kHz}$ directional source at $10 \mathrm{~m}$ depth ensonifying a single scatterer randomly located azimuthally on axis in a thin layer in a random waveguide (case F, Table II). The top two panels are for a scatterer in a shallow layer between 10 and $20 \mathrm{~m}$ depth, while the bottom two panels are for a scatterer in a deep layer between 85 and $95 \mathrm{~m}$ depth. The left two panels give the SI of the pressure field at the scatterer's random location, while the right two panels give the SI of the echoes at the receiver. The horizontal lines are the theoretical SI for a phase saturated pressure field $(\mathrm{SI}=1$ for the case of a Rayleigh distributed pressure magnitude at the scatterer location, and $\mathrm{SI}=5$ for exponential distributed echo magnitudes at the receiver). 
range-dependent effects seen in the intensity statistics of both the shallow and deep layers are illustrated (Fig. 13). First, the SI rapidly decays toward saturation in all cases (i.e., both shallow and deep layers and both at the target range and at the receiver). This trend toward saturation is in agreement with the results seen in Sec. V A where the trend toward saturation is largely driven by the increased randomness of the field with range due to the IW perturbations of the sound speed.

The second effect is that the SI shows a quasioscillating range structure that is dependent on the mean structure of the beam. That is, at ranges where the beam is weak in the layer (e.g., see Fig. 12, upward pointing arrows) SI is large and where the beam is strong in the layer (e.g., see Fig. 12, downward pointing arrows) SI is small. The peaks in the saturation index at short ranges can be largely attributed to the fact that the SI is normalized by the mean which has a tendency to elevate the SI in regions of very low pressure values. An analysis of the energetic region is more illuminating. In all cases the SI begins slightly to well below saturation (i.e., in an unsaturated state) and rises to or above saturation (i.e., a partially saturated state) before tending toward saturation.

In comparing the shallow and deep water cases it appears that the scattering from the deep layer approaches saturation at slightly shorter ranges. This is likely due to a difference in the structure and extent of the energetic zones. Near the surface the shape of this zone is influenced solely by the downward refracting SSP; whereas near the bottom there is a combination of effects due to both refraction within the water column and reflection from the bottom.

\section{B. Scatterer(s) in a 2D directional BP}

\section{Single scatterer}

In this section a final degree of complexity is added to the simulations: An azimuthally-dependent BP (see Table II, case G). A single scatterer, randomly located in the beam within a shallow layer $(10-20 \mathrm{~m})$ is first considered. Within these layers, the results are divided into energetic and nonenergetic (shadow zone) regions, horizontally, as denoted by the arrows (Fig. 12).

Echo magnitude PDFs were generated for various ranges from 1 to $9.125 \mathrm{~km}$. These results were compared with the PS calculation utilizing Eq. (16). Two parameters were adjusted to account for waveguide propagation effects: The range in DE of the BP and the amplitude of the scattering response. The random scatterer location in the beam is limited in $\mathrm{DE}, \Theta_{\mathrm{el}}$, to a range of angles centered on the main lobe, while the azimuthal angle, $\Theta_{\mathrm{az}}$, is given the full range within the half-space (i.e., the main lobe and all side lobes). Limiting the angle of DE represents the loss of acoustic energy that is highly attenuated by interaction with the bottom. Several ranges of DE were examined: The full beam ( $\pm \pi / 2$ radians), the main lobe and first side lobe, on axis ( \pm 0 radians), and a portion of the main lobe. Agreement with PE calculations was best when the angle of DE was limited to the angle where the source BP value first decreases to the amplitude of the highest side lobe, $\pm \theta_{\mathrm{el}, \mathrm{SL}}$. The waveguide-scatterer response, $\Upsilon_{n}$, is drawn from the square of a zero-mean complex Gaussian random variable (with a modulus that is exponentially distributed). This accounts for the squaring effect of the waveguide described in Sec. II C for a single scatterer in a saturated pressure field.

The PS PDFs are shown to be a very good estimate of the PE predictions at all ranges when the scatterer is located within the energetic zone and at long ranges for cases where the scatterer is located in the less energetic zone (Fig. 14). At short ranges, where the acoustic beam is very weak in the layer, the PS does not model the PDF well since the field is not saturated. However, as the layer becomes more uniformly ensonified with range, due to forward scattering by IWs, the PS predicts the PDF well $\left(d_{\mathrm{KL}}=3.82 \times 10^{-3}\right.$ at $9.125 \mathrm{~km})$.
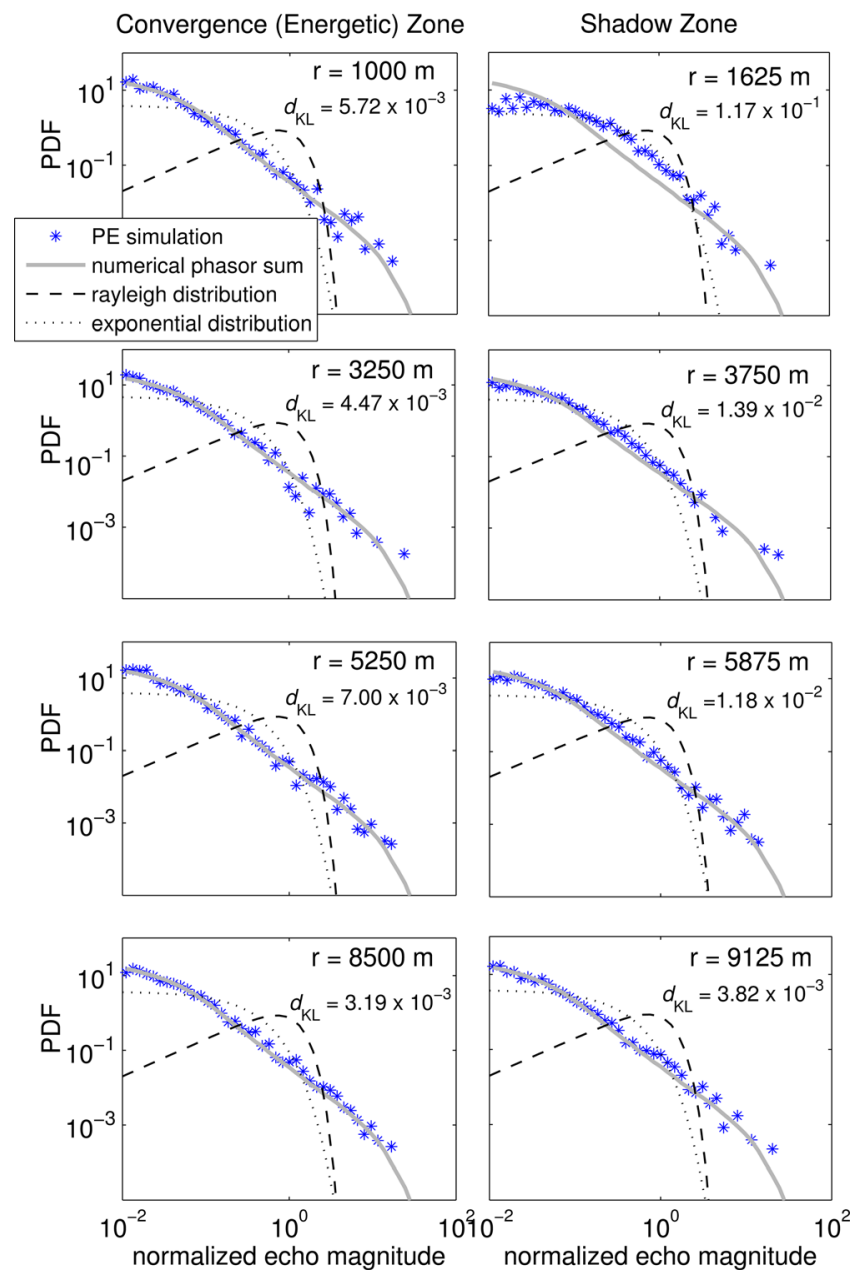

FIG. 14. (Color online) Predicted echo PDFs associated with a $3 \mathrm{kHz}$ directional source at a depth of $10 \mathrm{~m}$ ensonifying a single scatterer randomly located within the full $180^{\circ} \mathrm{BP}$, azimuthally, and in a shallow thin layer vertically (10-20 m), in a random waveguide (case G, Table II). The panels on the left give the statistics associated with the convergence zones and the panels on the right give the statistics of the shadow zones. The range of the scatterer, $r$, is given in the top right of each panel. The directional source is a circular piston $(k a=37.06)$ with a narrow beam $\left(5^{\circ}-3 \mathrm{~dB}\right.$ beam width $)$ and the scatterer response is a constant. Kullback-Leibler distances comparing PE predictions with phasor sum predictions, $d_{\mathrm{KL}}$, are given in the top right corner of each panel. 


\section{Multiple scatterers}

The simulation parameters in Sec. VI B 1 were applied at a single range for multiple scatterers assumed to have equal and fixed scattering responses. A range of $5.25 \mathrm{~km}$ was chosen where the echo statistics were determined to be near saturation $(N=1, \mathrm{SI}=5.22)$. The tails of the predicted distributions are strongly non-Rayleigh in all cases (Fig. 15). Notably, in the case of ten scatterers the result is still highly non-Rayleigh with a significantly elevated tail. Comparing the PE results with the PS shows excellent agreement for low values of $N\left(N \leq 5, d_{\mathrm{KL}} \leq 5.35 \times 10^{-3}\right)$ and good agreement in the tail at all values of $N$ studied. Comparisons were also made with the $K$ distribution using the method of moments to estimate the shape parameter. ${ }^{45}$ In all cases the physics-based PS method, not the $K$ distribution, is a better fit to the PE results.
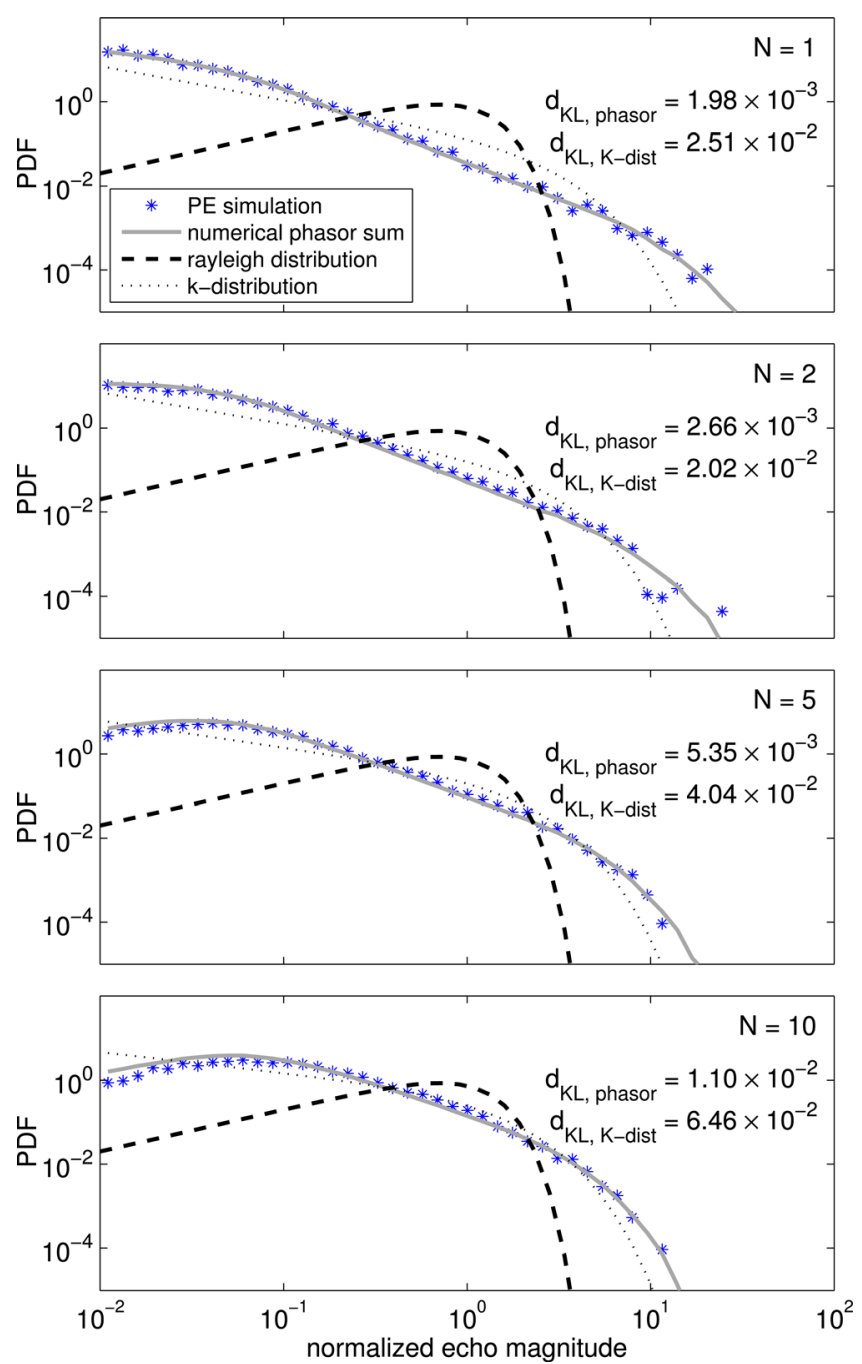

FIG. 15. (Color online) Predicted echo PDFs associated with a $3 \mathrm{kHz}$ directional source at a depth of $10 \mathrm{~m}$ ensonifying various numbers of scatterers with a constant scattering response. The scatterers are located within the full $180^{\circ} \mathrm{BP}$, azimuthally, and in a shallow thin layer vertically $(10-20 \mathrm{~m})$, at a range of $5250 \mathrm{~m}$ in a random waveguide (case G, Table II). The directional source is a circular piston $(k a=37.06)$ with a narrow beam $\left(5^{\circ}-3 \mathrm{~dB}\right.$ beam width). The number of scatterers, $N$, is given in the top right of each panel. Kullback-Leibler distances comparing PE predictions with phasor sum predictions, $d_{\mathrm{KL} \text {,phasor }}$, and the $K$ distribution, $d_{\mathrm{KL}, K-\text { dist }}$, are given in the top right corner of each panel.
The echo envelope distribution in the limit of many scatterers was determined by calculating the statistics for the case of $N=1000$ using the PS method. In this case the distribution of echo envelopes approaches a Rayleigh distribution $\left(d_{\mathrm{KL}}=1.87 \times 10^{-3}\right.$-figure not shown $)$.

\section{Extended, finite-sized Rayleigh scatterers}

The effects of including a scatterer with a realistic scattering response and dimensions were investigated (see Table II, case $\mathrm{H}$ ) by simulating a Rayleigh scatterer of finite extent with a patch of 10 point scatterers located closely in space within a sphere with a $2.5 \mathrm{~m}$ radius. Predictions in Sec. IV D suggest that this geometry provides a spatial extent sufficient to induce a Rayleigh-like scattering response [Fig. 9 (bottom two panels)]. In all cases, for $N$ ranging from 1 to 10 , the PDFs of the echo
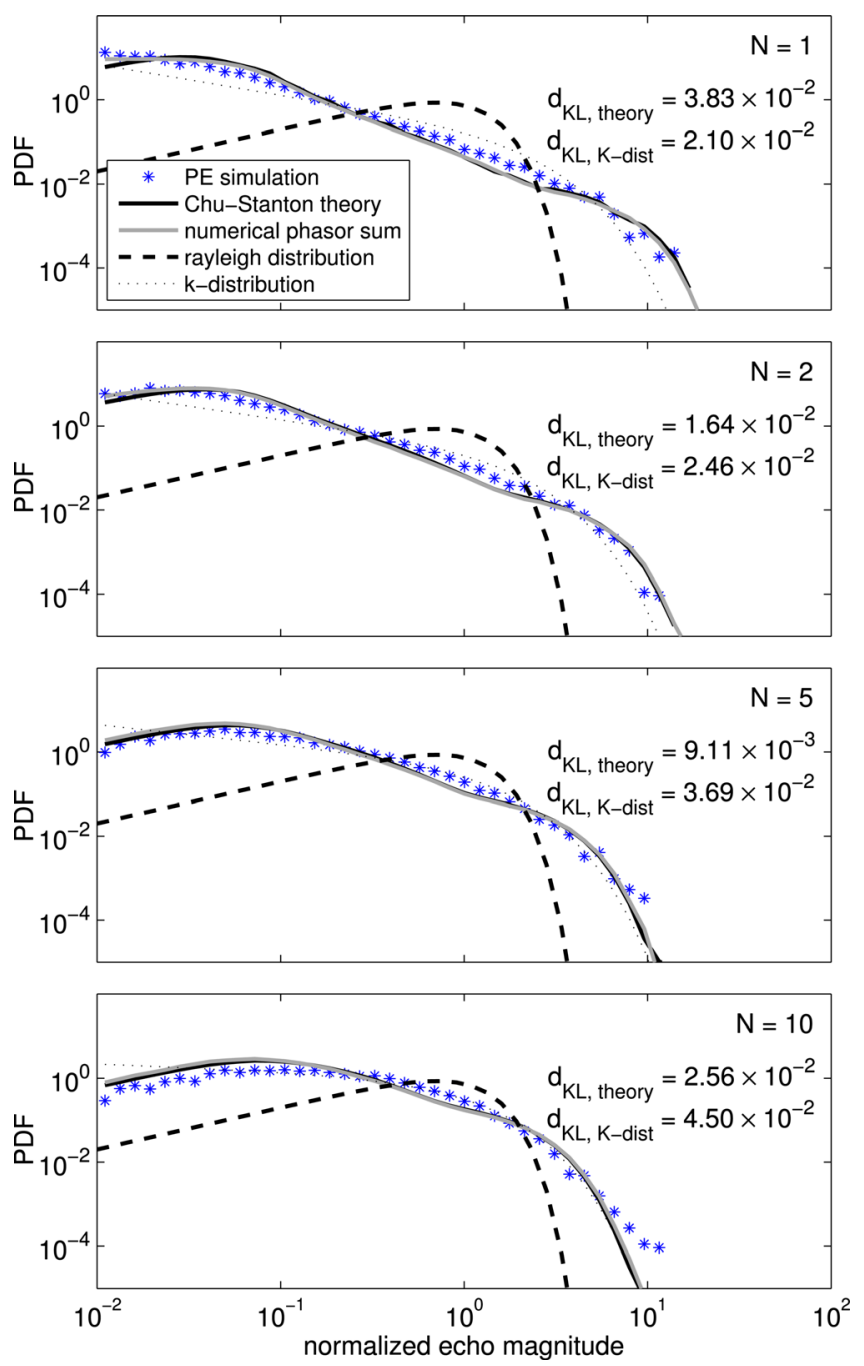

FIG. 16. (Color online) Predicted echo PDFs associated with a $3 \mathrm{kHz}$ directional source at a depth of $10 \mathrm{~m}$ ensonifying various numbers of extended Rayleigh scatterers. The scatterers are located within the full $180^{\circ} \mathrm{BP}$, azimuthally, and in a thin layer, vertically, at a range of $5250 \mathrm{~m}$ in a random oceanic waveguide (case H, Table II). The directional source is a circular piston $(k a=37.06)$ with a narrow beam $\left(5^{\circ}-3 \mathrm{~dB}\right.$ beam width). The number of scatterers, $N$, is given in the top right of each panel. Kullback-Leibler distances comparing PE predictions with the waveguide-modified Chu-Stanton theory, $d_{\mathrm{KL} \text {,theory }}$, and the $K$ distribution, $d_{\mathrm{KL}, K-\text { dist }}$, are given in the top right corner of each panel. 
magnitudes are strongly non-Rayleigh (Fig. 16). The trend of the PDFs with increasing $N$ is, for both the low and high amplitude ends of the PDF, to decrease toward a Rayleigh distribution. Comparisons are made with the analytical form of the Chu-Stanton theory modified to include waveguide effects (Sec. IID) as well as the mathematically equivalent numerical PS.

In these calculations the angles of DE of the scatterers, $\Theta_{\mathrm{el}}$, are limited to $\pm \theta_{\mathrm{el}, \mathrm{SL}}$ as in the comparisons with the delta-function scatterers. However, the scattering amplitude, $\Upsilon_{n}$, is drawn from a zero-mean complex Gaussian distribution (with a modulus that is Rayleigh distributed) to model the scattering response of large, extended Rayleigh scatterers in a waveguide (see Sec. IV D). There is good agreement in all cases $\left(d_{\mathrm{KL}} \leq 3.83 \times 10^{-2}\right)$. In the case of $N=10$, there is not as good agreement in the tail of the PDF; however, the theory still performs similarly or better than the $K$ distribution in all cases.

Calculations using the PS method were also made to investigate the echo envelope statistics in the limit of many scatterers. In this case of $N=1000$ the distribution of echo envelopes approaches a Rayleigh distribution $\left(d_{\mathrm{KL}}=2.38\right.$ $\times 10^{-3}$-figure not shown).

Fits of the $K$ distribution to the simulations show that the shape parameter, $\alpha_{k}$, often used as a proxy for the number of scatterers in a sonar resolution cell, ${ }^{31,39,40,62}$ does not follow a one-to-one relationship with the effective number of scatterers, $N_{\text {eff }}$ (Fig. 17). This effective number of scatterers is the product of the total number of scatterers in the half space and the fractional width of the BP. Although $\alpha_{\mathrm{k}}$ shows a linear relationship with $N$ there is a significant offset from the origin at $N=0$ suggesting more information is needed than just the number of scatterers to predict a $K$ distribution that fits the echo envelopes.

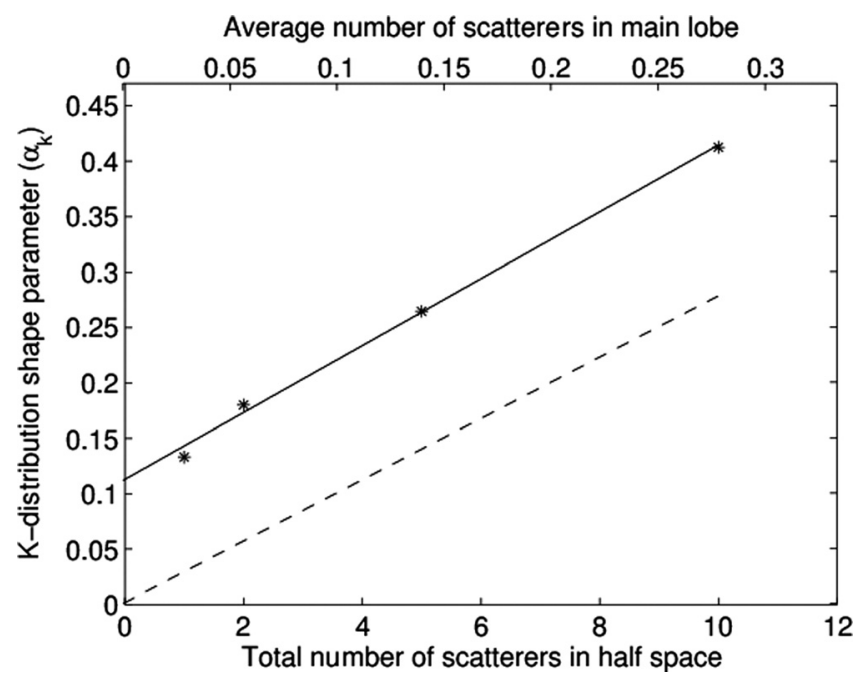

FIG. 17. $K$ distribution shape parameter, $\alpha_{k}$, as a function of the number of scatterers ensonified by a directional beam in a shallow water oceanic waveguide. The simulation is the same as that described in the Fig. 16 (case H, Table II). The black asterisks are the best fit shape parameter versus the number of scatterers located in the half-space. The solid line is a linear fit to the data. The dashed line is $\alpha_{k}$ equal to the average number of scatterers per main lobe $\left(5^{\circ}-3 \mathrm{~dB}\right.$ beam width $)$.

\section{DISCUSSION}

Echo statistics for a monostatic, single frequency sonar system have been studied in terms of the characteristics of the sonar system, the waveguide, and properties of the scatterers. Each of these components significantly affects the statistics and their separate contributions are summarized below.

\section{A. Effects of a deterministic waveguide}

In a deterministic waveguide, a statistical distribution of echo envelopes can result from a random spatial distribution of scatterers in the water column. Of critical importance is how the random placement of scatterers coincides with the acoustic interference pattern and/or shadow zones of the forward-propagated field, the characteristics of which are determined by boundary interactions, refraction, and the directionality of the source. In the case where there is no shadow zone and the interference pattern is the result of many multipaths, such as a result from an omnidirectional source or weakly refracting SSP, the envelope PDF of the one-way pressure-field will trend toward a Rayleigh with echo envelopes that, for a single scatterer, trend toward an exponential PDF. Factors which reduce this trend by inhibiting multipath interference include large bottom attenuation, strongly refracting profiles, and a strongly directional source.

There are two salient observations from these results. First, a strong deterministic multipath can produce a complex-Gaussian distributed pressure field (i.e., a Rayleigh-distributed envelope after one-way propagation) when sampled randomly in space at a fixed range. This result is statistically equivalent to a saturated pressure field associated with a stochastic process. Second, the PDF of echo envelopes (after two-way propagation) for a single scatterer randomly located in a Rayleigh-distributed forward field trends not toward a Rayleigh distribution, but toward an exponential due to squaring effect of the (frozen) waveguide. We believe this latter result has not been previously described in the literature.

\section{B. Effects of a random waveguide for one or more scatterers}

Adding IW-induced sound-speed perturbations enhances multipath interference and increases ensonification of the shadow zones. Both of these effects increase the trend toward Rayleigh statistics (saturation) for one-way propagation. The effect of enhanced multipath on the statistics of the echoes after two-way propagation is best illustrated by comparing Figs. 8 and 10 (right panels). For a directional acoustic source in a strongly refracting environment, the addition of IW-induced sound-speed fluctuations (case E) causes a trend toward exponentially distributed echo envelopes for a randomly located scatterer $(N=1)$ in the energetic region of the waveguide (Fig. 10). This is not seen in the case of a range-independent sound-speed field (case D, Fig. 8) which shows very little range dependence beyond $3.5 \mathrm{~km}$. While the channeling of acoustic energy away from the boundaries 
may limit deterministic multipath formation, and thus interference, the presence of stochastic sound-speed structure may induce sufficient multipath interference to drive the acoustic field to saturation at relatively short ranges.

The combined effect of reduced shadow zones and increasing multipath is seen in the case of thin layers of scatterers ensonified by a shallow source in a stochastic waveguide (case F). At short ranges, the SI is strongly range-dependent (Fig. 13), driven by the location of the scatterers with respect to the alternating energetic and shadow regions. These regions are generally deterministic and are formed by the refraction of the narrow acoustic beam as it is influenced by the mean SSP. However, forward scattering by IWs increasingly scatterers energy into the shadow zone with range (Fig. 12) and the SI converges on saturation values both for the one-way path $(\mathrm{SI}=1)$ and for the echoes at the receiver $(\mathrm{SI}=5)$.

Diffuse IWs are just one source of random fluctuation in the ocean. Other stochastic processes include eddies and associated filaments, nonlinear IWs, and spice (i.e., density compensated thermohaline structure). It is expected that other stochastic waveguide processes such as these would have statistical similarities to the processes represented in this study in that they would increase the phase and amplitude variation of the pressure field and, potentially, induce saturation of the pressure field at even shorter ranges. In general, the stochastic components of the waveguide simplify the prediction of echo envelope distributions for horizontal looking systems by removing the range dependence of the waveguide effects at ranges beyond the point where saturation is reached. However, other factors may still contribute a range-dependent effect on the echo statistics, such as number of scatterers in the sonar beam which can vary with range.

When multiple, independent scatterers are ensonified by a vertically directional source in a random waveguide the echo envelope PDF trends back toward a Rayleigh distribution (e.g., Fig. 11). Thus, for a saturated or nearly saturated pressure field at the scatterer, and no azimuthal BP effects, the echo envelope distribution is bounded by the exponential distribution for small $N$ and a Rayleigh distribution for large $N$. However, while the distributions are clearly approaching a Rayleigh with increasing $N$, the echo PDF, even in the case of ten scatterers, still has a significantly elevated tail with respect to a Rayleigh PDF. Furthermore, in the case where the beam is narrow in both DE and azimuth the trend toward a Rayleigh distribution with increasing $N$ is even more gradual (Fig. 15).

\section{Effects of a directional source}

The elevation-dependent component of source directionality (in the vertical plane) was shown to slow the progress toward saturation of the forward field by producing shadow zones and limiting boundary interactions. The azimuthal component (in the horizontal plane) was shown to influence the echo statistics directly by non-uniformly ensonifying scatterers that are wholly within the energetic zone. This latter characteristic of the sonar system can lead to nonexponential echo envelope PDFs for the case of a single scatterer and non-Rayleigh statistics even for a relative large number of scatterers located in a saturated forward field. In this study the effects of the azimuthal component are explored in the full BP simulations (cases $\mathrm{G}$ and $\mathrm{H}$ ).

The azimuthal component of the BP has the strong and consistent effect of inducing an elevated, high-amplitude "tail" to the echo-envelope distribution for scatterers located at all ranges (illustrated by the full BP cases $\mathrm{G}$ and $\mathrm{H}$ ). Importantly, the combination of a directional sonar beam and a single delta-function scatterer $(N=1)$, located in a randomized waveguide, produces a highly non-exponential (and non-Rayleigh) echo PDF (Fig. 14). Note, though, that out-of-plane scattering has been ignored in these simulations. It is expected that strong horizontal variations in the SSP would lessen the effect of the azimuthal BP component.

\section{Extended scatterers with a variable scattering response (Rayleigh scatterer) in a waveguide}

Much of the focus of this study has been on scatterers with fixed responses; however, in nature the scattering response is often variable. One commonly observed response is a complex Gaussian distribution which arises from a patch of many scatterers, with similar scattering amplitudes and random phase, contributing to a single echo (e.g., a compact school of single-sized fish ensonified near swim bladder resonance). This response induces echo envelopes that are Rayleigh distributed in the absence of waveguide or BP effects and leads to the term Rayleigh scatterer to describe the patch. This study shows that the size of such a patch in a waveguide determines whether the echo envelopes are nonRayleigh distributed (small scatterers) or Rayleigh distributed (large scattering volumes-before BP effects are included).

A Rayleigh scatterer in a waveguide with a zero-mean complex-Gaussian pressure field (statistically equivalent to a saturated environment) is examined in case B. While an infinitesimally small Rayleigh scatterer (i.e., a point scatterer with a complex Gaussian response) in a waveguide has a highly non-Rayleigh echo envelope distribution, the echo from an extended Rayleigh scatterer of this nature has an increasingly Rayleigh-like distribution with increasing patch size (Fig. 9). This result is further validated in case $\mathrm{H}$, where an extended Rayleigh scatterer is modeled in the saturated pressure field of a random waveguide giving predictable results (Fig. 16).

The reason for this dependency on the scatterer size, shown mathematically in Sec. IIE, is related to the relative size of the patch with respect to the distance at which the one-way pressure field becomes uncorrelated. For a very small scatterer the amplitude of the entire scattering volume is modulated by the waveguide response, which is exponentially distributed. However, in the limit of a large patch size, different regions of the scattering volume are modulated independently, further randomizing the contribution from various regions of the scattering volume. Following the CLT then, the summation of the contributions from various regions of scattering volume results in echo envelopes that are Rayleigh distributed. In practice, the ensonified volume 
TABLE III. Predicted echo envelope PDFs for various cases of a monostatic sonar system with $N$ scatterers positioned randomly in the saturated pressure field of a waveguide.

Without beam pattern effects

\begin{tabular}{cccc}
\hline$N$ & Scatterer response & Spatial extent & PDF \\
\hline 1 & delta & point & exponential \\
1 & Rayleigh & point & product PDF $^{\text {a }}$ \\
1 & Rayleigh & extended & Rayleigh (in limit of large spatial extent)
\end{tabular}

With beam pattern effects

\begin{tabular}{|c|c|c|c|c|c|}
\hline$N$ & Scattering response & Scatterer spatial extent & Elevation-dependent BP & Azimuthally dependent $\mathrm{BP}^{\mathrm{b}}$ & PDF \\
\hline 1 & delta & point & yes & no & exponential \\
\hline$\gg 1$ & delta & point & yes & no & approaches Rayleigh $\mathrm{O}(N \approx 10)$ \\
\hline 1 & delta & point & yes & yes & highly elevated tail ${ }^{c}$ \\
\hline$\gg 1$ & delta & point & yes & yes & approaches Rayleigh $\mathrm{O}(N \approx 1000)$ \\
\hline 1 & Rayleigh & extended & yes & yes & highly elevated tail $^{\mathrm{c}}$ \\
\hline$\gg 1$ & Rayleigh & extended & yes & yes & approaches Rayleigh $\mathrm{O}(N \approx 1000)$ \\
\hline
\end{tabular}

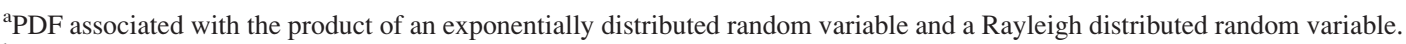

${ }^{\mathrm{b}}$ No out-of-plane scattering considered.

${ }^{\mathrm{c}}$ Distribution with high-amplitude portion elevated above Rayleigh and exponential distributions.

that contributes to a single echo is dependent on the sonar source and receiver beam widths and the source waveform. This dependence on the size of the scattering volume is only expected while the scatterer is smaller than the spatial resolution of the sonar system.

These results have important implications for modeling compact aggregations of scatterers in the water column. It is important to model an extended, finite-size Rayleigh scatterer with a representative spatial extent rather than by a single point scatterer with a non-uniform scattering response (e.g., the Rayleigh, point target in Fig. 9) as the echo statistics of the two cases can be significantly different.

\section{E. Model comparisons}

A key result of this analysis is that the echo statistics of a collection of isotropic scatterers in a complex oceanic environment may be modeled in some cases with a fairly simple and computationally efficient model such as the PS method described in Sec. III C. The requirements are that the scatterers are located in an energetic zone of the waveguide and at a range where the one-way pressure field is saturated. Knowledge then is required of the BP, the number of scatterers in the beam at a given range, and the scattering response (e.g., constant or complex Gaussian).

In nearly all cases studied, both the Chu-Stanton theory and the mathematically equivalent PS method outperformed the $K$ distribution in predicting echo statistics from water column scatterers in a shallow waveguide (Figs. 15 and 16). Furthermore, it is shown that in the case of $N$ scatterers randomly distributed in a directional beam, the shape parameter of a $K$ distribution that most closely fits the data cannot be predicted solely from the number of ensonified scatterers (Fig. 17).

\section{CONCLUSIONS}

In conclusion, the degree to which echo statistics are non-Rayleigh has been quantified in terms of a range and a combination of properties of the waveguide, sonar system, and scatterers. Parameters shown to strongly influence the echo PDFs are randomness of the waveguide, directionality of the sonar beam, and properties of the scatterer including the number present, scattering response, and spatial extent. Key combinations of these various properties and the resulting echo PDFs are summarized in Table III. Quantification of these dependencies represent advancements through realistic modeling of the spatially coherent, random waveguide properties, BP effects, and spatial extents of aggregations of scatterers.

Several models of varying complexity were used in the analysis. While the two-way PE model incorporating realistic IW fields was the most rigorous, it also required significant computational time. A simple PS method was shown to be very effective and efficient over a range of important conditions. Finally, the PS method and Chu-Stanton theory were shown to outperform the $K$-distribution in cases involving a directional BP.

\section{ACKNOWLEDGMENTS}

This research was funded, in part, by the Office of Naval Research Grant Nos. N00014-10-1-0127 and N0001411-1-0241 and the Oceanographer of the U.S. Navy. The authors would like to thank Dr. J. Paquin Fabre of the Naval Research Laboratory, Stennis Space Center, for sharing her MATLAB-based PE code which was modified for use in this study. We also wish to thank Dr. Ching-Sang Chiu, Dr. Lawrence Ziomeck, Dr. Patrick Harr, and Dr. D. Benjamin Reeder at the Naval Postgraduate School for their inputs throughout this research process.

\section{APPENDIX A: GENERATION AND COMPARATIVE ANALYSIS OF PDFS OF ECHO ENVELOPES}

Statistical ensembles were generated by the Monte Carlo method with an ensemble size of at least 1000 realizations in all cases which was shown to produce good results 
for the two-way PE model in validation against theory (see Sec. III E). PDFs were generated from histograms of envelopes normalized by the rms echo amplitude of the ensemble. A subtle consequence of this normalization process is that the single-parameter Rayleigh distribution, with a parameter that is directly related to the rms value, will have a fixed probability distribution (i.e., no free parameters) when normalized. In contrast the exponential distribution, which has a single parameter representing the mean of the distribution, will vary from normalization to normalization.

Two statistical methods were used in this paper to compare PDFs, the Lilliefors test and the Kullback-Leibler distance. The Lilliefors test is a goodness-of-fit test used to determine if a given distribution comes from a specific distribution in the normal family. ${ }^{63,64}$ The two primary distributions of interest in this study are the Rayleigh distribution and the exponential distribution. The Lilliefors test is used to test goodness-of-fit for the exponential distribution directly. For the Rayleigh distribution, which arises when the modulus is taken of a zero-mean Gaussian random variable, the Lilliefors test is applied independently to the real and imaginary parts of the complex pressure before taking the envelope. These results are presented as $p$-values for both the real and imaginary parts of the complex random variable (e.g., $p_{\text {real }} / p_{\text {imag. }}=0.8 / 0.9$ ) represents the $p$-value of 0.8 for the real portion and 0.9 for the imaginary portion for the goodness-of-fit to a Gaussian distribution. In this case the high $p$ values would indicate that the distribution is Rayleigh distributed. The Kullback-Leibler distance provides a statistical measure of the difference between two distributions. ${ }^{65}$ This measure is given as

$$
d_{\mathrm{KL}}=\sum_{i} \mathrm{p}_{1}\left(x_{i}\right) \log \left[\frac{\mathrm{p}_{1}\left(x_{i}\right)}{\mathrm{p}_{2}\left(x_{i}\right)}\right]
$$

where the probabilities $\mathrm{p}_{1}(\cdot)$ and $\mathrm{p}_{2}(\cdot)$ can represent either a model prediction and a theoretical distribution or two model predictions. This measure emphasizes the mismatch between the high amplitude, low probability tails of the distributions. ${ }^{46}$

\section{APPENDIX B: CHARACTERIZATION OF ECHOES USING SCINTILLATION INDEX}

The SI is the normalized intensity variance of the randomly sampled intensity, $I$, given as

$$
\mathrm{SI}=\frac{\left\langle I^{2}\right\rangle}{\langle I\rangle^{2}}-1 .
$$

It is known that the SI of a fully saturated (i.e., complex Gaussian) pressure field is $1 ;^{66}$ however, the SI may also be used to analyze the statistics of echoes measured at a receiver. Given a narrowband signal in a strong multipath environment, the pressure at a random position and given time can be described by the sum of the contributions from $k$ individual paths, $P(\vec{R}, t)=\sum_{k} A_{k} e^{i \Phi_{k}}$, with amplitude $A_{k}$ and phase $\Phi_{k}$. It has been shown ${ }^{41}$ that, when $\left\langle\Phi^{2}\right\rangle \gg 1$, Eq. (B1) can be expressed in terms of the individual path amplitude, $A$, as

$$
\mathrm{SI}=1+\frac{1}{N}\left(\frac{\left\langle A^{4}\right\rangle}{\left\langle A^{2}\right\rangle^{2}}-2\right)
$$

For the case of a backscattered signal from multiple scatterers, the contribution at the receiver from each scatterer can be treated as a separate path. It is seen in this study that, for the case of a single scatterer $(N=1)$ in a saturated pressure field, the echo magnitude statistics at the receiver have an exponential distribution. Given then that the $n$th moment of the echo amplitude is described by $\left\langle A^{n}\right\rangle=n !\langle A\rangle^{n}$, substituting into Eq. (B2) gives

$$
\mathrm{SI}=1+4 / N \text {. }
$$

${ }^{1}$ D. E. Weston and J. Revie, "Fish echoes on a long-range sonar display," J. Sound Vib. 17, 105-112 (1971).

${ }^{2}$ J. S. M. Rusby, M. L. Somers, J. Revie, B. S. McCartney, and A. R. Stubbs, "Experimental survey of a herring fishery by long-range sonar," Mar. Biol. 22, 271-292 (1973).

${ }^{3}$ D. E. Weston and H. W. Andrews, "Seasonal sonar observations of the diurnal shoaling times of fish,” J. Acoust. Soc. Am. 87, 673-680 (1990).

${ }^{4}$ D. M. Farmer, M. V. Trevorrow, and B. Pedersen, "Intermediate range fish detection with a 12-kHz sidescan sonar," J. Acoust. Soc. Am. 106, 2481-2490 (1999).

${ }^{5}$ N. C. Makris, P. Ratilal, D. T. Symonds, S. Jagannathan, S. Lee, and R. W. Nero, "Fish population and behavior revealed by instantaneous continental shelf-scale imaging," Science 311, 660-663 (2006).

${ }^{6}$ T. K. Stanton, J. M. Jech, R. C. Gauss, B. A. Jones, C. J. Sellers, and J. M. Fialkowski, "Observations of region-specific fish behavior using long- and short-range broadband (1.5-6+ kHz) active acoustic systems," J. Acoust. Soc. Am. 134, 3989 (2013).

${ }^{7}$ Y. A. Kravtsov, V. M. Kuz'kin, and V. G. Petnikov, "Wave diffraction by regular scatterers in multimode waveguides," Sov. Phys. Acoust. 30, 199-202 (1984).

${ }^{8}$ F. Ingenito, "Scattering from an object in a stratified medium," J. Acoust. Soc. Am. 82, 2051-2059 (1987).

${ }^{9}$ A. Boström, "Transmission and reflection of acoustic waves by an obstacle in a waveguide," Wave Motion 2, 167-184 (1980).

${ }^{10}$ R. H. Hackman and G. S. Sammelmann, "Acoustic scattering in an inhomogeneous waveguide: Theory," J. Acoust. Soc. Am. 80, 1447-1458 (1986).

${ }^{11}$ R. H. Hackman and G. S. Sammelmann, "Long-range scattering in a deep oceanic waveguide," J. Acoust. Soc. Am. 83, 1776-1793 (1988).

${ }^{12}$ R. Lim and R. H. Hackman, "A formulation of multiple scattering by many bounded obstacles using a multicentered, $T$ supermatrix," J. Acoust. Soc. Am. 91, 613-638 (1992).

${ }^{13}$ M. D. Collins and M. F. Werby, "A parabolic equation model for scattering in the ocean," J. Acoust. Soc. Am. 85, 1895-1902 (1989).

${ }^{14}$ G. S. Sammelmann and R. H. Hackman, "Acoustic scattering in a homogeneous waveguide,” J. Acoust. Soc. Am. 82, 324-336 (1987).

${ }^{15}$ M. Tran-Van-Nhieu, "Scattering from slender bodies in a homogeneous waveguide," J. Acoust. Soc. Am. 88, 2880-2888 (1990).

${ }^{16}$ L.-W. Cai, D. K. Dacol, D. C. Calvo, and G. J. Orris, "Acoustical scattering by arrays of cylinders in waveguides," J. Acoust. Soc. Am. 122, 1340-1351 (2007).

${ }^{17}$ G. V. Norton and M. F. Werby, "A numerical technique to describe acoustical scattering and propagation from an object in a waveguide," J. Appl. Phys. 70, 4101-4112 (1991).

${ }^{18}$ J. A. Fawcett, "A plane-wave decomposition method for modeling scattering from objects and bathymetry in a waveguide," J. Acoust. Soc. Am. 100, 183-192 (1996).

${ }^{19} \mathrm{R}$. Lim, "Multiple scattering by many bounded obstacles in a multilayered acoustic medium," J. Acoust. Soc. Am. 92, 1593-1612 (1992).

${ }^{20}$ D. K. Dacol and D. G. Roy, "The partial-wave expansion for scattering in waveguides," J. Acoust. Soc. Am. 120, 2518-2525 (2006).

${ }^{21}$ E. T. Küsel and P. Ratilal, "Effects of incident field refraction on scattered field from vertically extended cylindrical targets in range-dependent ocean waveguides," J. Acoust. Soc. Am. 125, 1930-1936 (2009). 
${ }^{22}$ J. A. Fawcett, "Modeling acousto-elastic waveguide/object scattering with the Rayleigh hypothesis," J. Acoust. Soc. Am. 106, 164-168 (1999).

${ }^{23} \mathrm{~W}$. H. Munk and F. Zachariasen, "Sound propagation through a fluctuating stratified ocean: Theory and observation," J. Acoust. Soc. Am. 59, 818-838 (1976).

${ }^{24}$ S. M. Flatté, Sound Transmission Through a Fluctuating Ocean (Cambridge University Press, Cambridge, 1979), Chaps. 6-9.

${ }^{25}$ M. Andrews, Z. Gong, and P. Ratilal, "Effects of multiple scattering, attenuation and dispersion in waveguide sensing of fish," J. Acoust. Soc. Am. 130, 1253-1271 (2011).

${ }^{26}$ S. Jagannathan, E. T. Küsel, P. Ratilal, and N. C. Makris, "Scattering from extended targets in range-dependent fluctuating ocean-waveguides with clutter from theory and experiments," J. Acoust. Soc. Am. 132, 680-693 (2012).

${ }^{27}$ D. Chu and T. K. Stanton, "Statistics of echoes from a directional sonar beam insonifying finite numbers of single scatterers and patches of scatterers," IEEE J. Ocean. Eng. 35, 267-277 (2010).

${ }^{28} \mathrm{~K}$. D. LePage, "Statistics of broad-band bottom reverberation predictions in shallow-water waveguides," IEEE J. Ocean. Eng. 29, 330-346 (2004).

${ }^{29}$ T. C. Weber, H. Pena, and J. M. Jech, "Consecutive acoustic observations of an Atlantic herring school in the Northwest Atlantic," ICES J. Mar. Sci. 66, 1270-1277 (2009).

${ }^{30}$ S. Bhatia, T. K. Stanton, and K. B. Baik, "Non-Rayleigh scattering by a randomly oriented elongated scatterer randomly located in a beam," IEEE J. Ocean. Eng. in press (2014), DOI: 10.1109/JOE.2013.2293959.

${ }^{31}$ D. A. Abraham, "The effect of multipath on the envelope statistics of bottom clutter," IEEE J. Ocean. Eng. 32, 848-861 (2007).

${ }^{32}$ R. W. Nero, C. H. Thompson, and J. M. Jech, "In situ acoustic estimates of the swim bladder volume of Atlantic herring (Clupea harengus)," ICES J. Mar. Sci. 61, 323-337 (2004).

${ }^{33}$ T. K. Stanton, C. J. Sellers, and J. M. Jech, "Resonance classification of mixed assemblages of fish with swimbladders using a modified commercial broadband acoustic echosounder at $1-6 \mathrm{kHz}$," Can. J. Fish. Aquat. Sci. 69, 854-868 (2012).

${ }^{34} \mathrm{~B}$. A. Jones, "Echo statistics of aggregations of scatterers in a random waveguide: Application to biologic sonar clutter." Ph.D. dissertation, Naval Postgraduate School (2012).

${ }^{35}$ B. F. Cron and W. R. Schumacher, "Theoretical and experimental study of underwater sound reverberation," J. Acoust. Soc. Am. 33, 881-888 (1961).

${ }^{36}$ D. A. Abraham and A. P. Lyons, "Guest editorial: Non-Rayleigh reverberation and clutter," IEEE J. Ocean. Eng. 29, 193-196 (2004).

${ }^{37}$ J. M. Gelb, R. E. Heath, and G. L. Tipple, "Statistics of distinct clutter classes in midfrequency active sonar," IEEE J. Ocean. Eng. 35, 220-229 (2010).

${ }^{38}$ T. K. Stanton and C. S. Clay, "Sonar echo statistics as a remote-sensing tool: Volume and sea-floor," IEEE J. Ocean. Eng. 11, 79-96 (1986).

${ }^{39}$ D. A. Abraham and C. W. Holland, "Spatially compact clutter," in Proceedings of 2008 International Symposium on Underwater Reverberation and Clutter (2008), pp. 247-254.

${ }^{40}$ D. A. Abraham and A. P. Lyons, "Novel physical interpretations of $K$ distributed reverberation," IEEE J. Ocean. Eng. 27, 800-813 (2002).

${ }^{41}$ J. A. Colosi and A. B. Baggeroer, "On the kinematics of broadband multipath scintillation and the approach to saturation," J. Acoust. Soc. Am. 116, 3515-3522 (2004).

${ }^{42}$ J. E. Ehrenberg, "A method for extracting the fish target strength distribution from acoustic echoes," in Proceedings of the International Conference on Engineering in the Ocean Environment, Ocean 72 (1972), pp. 61-64.

${ }^{43}$ J. E. Ehrenberg, T. J. Carlson, J. J. Traynor, and N. J. Williamson, "Indirect measurement of the mean acoustic backscattering cross-section of fish,” J. Acoust. Soc. Am. 69, 955-962 (1981).

${ }^{44}$ R. Barakat, "First-order statistics of combined random sinusoidal waves with applications to laser speckle patterns," Opt. Acta 21, 903-921 (1974).
${ }^{45}$ D. A. Abraham and A. P. Lyons, "Reliable methods for estimating the $K$-distribution shape parameter," IEEE J. Ocean. Eng. 35, 288-302 (2010).

${ }^{46}$ T. K. Stanton and D. Chu, "Non-Rayleigh echoes from resolved individuals and patches of resonant fish at 2-4 kHz," IEEE J. Ocean. Eng. 35, 152-163 (2010).

${ }^{47}$ M. J. Mills, M. D. Collins, and J. F. Lingevitch, "Two-way parabolic equation techniques for diffraction and scattering problems," Wave Motion 31, 173-180 (2000).

${ }^{48}$ M. D. Collins, "A split-step Padé solution for the parabolic equation method," J. Acoust. Soc. Am. 93, 1736-1742 (1993).

${ }^{49}$ M. Strasberg, "Gas bubbles as sources of sound in liquids," J. Acoust. Soc. Am. 28, 20-26 (1956).

${ }^{50}$ F. B. Jensen, W. A. Kuperman, M. B. Porter, and H. Schmidt, Computational Ocean Acoustics (Springer-Verlag, New York, 2000), p. 348.

${ }^{51}$ J. A. Colosi, T. F. Duda, Y. T. Lin, J. F. Lynch, A. E. Newhall, and B. D. Cornuelle, "Observations of sound-speed fluctuations on the New Jersey continental shelf in the summer of 2006," J. Acoust. Soc. Am. 131, 1733-1748 (2012).

${ }^{52} \mathrm{~W}$. Munk, "Internal waves and small scale processes," in The Evolution of Physical Oceanography: Scientific Surveys in Honor of Henry Stommel, edited by B. A. Warren and C. Wunsch (MIT Press, Cambridge, 1981), pp. 264-291.

${ }^{53}$ W. H. Munk, "Sound channel in an exponentially stratified ocean, with application to SOFAR,” J. Acoust. Soc. Am. 55, 220-226 (1974).

${ }^{54}$ J. A. Colosi and M. G. Brown, "Efficient numerical stimulation of stochastic internal-wave-induced sound-speed perturbation fields," J. Acoust. Soc. Am. 103, 2232-2235 (1998).

${ }^{55}$ J. A. Colosi, T. F. Duda, and A. K. Morozov, "Statistics of low-frequency normal-mode amplitudes in an ocean with random sound-speed perturbations: Shallow-water environments," J. Acoust. Soc. Am. 131, 1749-1761 (2012).

${ }^{56}$ M. F. Arndt, "A method for generating uniform point distributions when the volume integral of a sample has constant integration limits in some curvilinear coordinate system," Nucl. Instrum. Method A 580, 1474-1477 (2007).

${ }^{57}$ S. M. Flatté and F. D. Tappert, "Calculation of the effect of internal waves on oceanic sound transmission," J. Acoust. Soc. Am. 58, 1151-1159 (1975).

${ }^{58}$ J. A. Colosi and A. K. Morozov, "Statistics of normal mode amplitudes in an ocean with random sound-speed perturbations: Cross-mode coherence and mean intensity," J. Acoust. Soc. Am. 126, 1026-1035 (2009).

${ }^{59}$ L. J. Van Uffelen, P. F. Worcester, M. A. Dzieciuch, and D. L. Rudnick, "The vertical structure of shadow-zone arrivals at long range in the ocean," J. Acoust. Soc. Am. 125, 3569-3588 (2009).

${ }^{60}$ L. J. Van Uffelen, P. F. Worcester, M. A. Dzieciuch, D. L. Rudnick, and J. A. Colosi, "Effects of upper ocean sound-speed structure on deep acoustic shadow-zone arrivals at 500- and 1000-km range," J. Acoust. Soc. Am. 127, 2169-2181 (2010).

${ }^{61}$ J. W. Goodman, Statistical Optics (John Wiley and Sons, New York, 1985), pp. 44-46.

${ }^{62}$ D. A. Abraham and A. P. Lyons, "Reverberation envelope statistics and their dependence on sonar bandwidth and scattering patch size," IEEE J. Ocean. Eng. 29, 126-137 (2004).

${ }^{63} \mathrm{H}$. W. Lilliefors, "On Kolmogorov-Smirnov test for normality with mean and variance unknown," J. Am. Stat. Assoc. 62, 399-402 (1967).

${ }^{64} \mathrm{H}$. W. Lilliefors, "On Kolmogorov-Smirnov test for exponential distribution with mean unknown," J. Am. Stat. Assoc. 64, 387-389 (1969).

${ }^{65}$ S. Kullback and R. A. Leibler, "On information and sufficiency," Ann. Math. Stat. 22, 79-86 (1951).

${ }^{66}$ I. Dyer, "Statistics of sound propagation in the ocean," J. Acoust. Soc. Am. 48, 337-345 (1970). 